\title{
Attenuation and velocity of elastic waves in polycrystals with generally anisotropic grains: analytic and numerical modeling
}

\author{
G. Sha ${ }^{\mathrm{a}}$, M. Huang ${ }^{\mathrm{b}}$, M. J. S. Lowe ${ }^{\mathrm{b}}$, S. I. Rokhlin ${ }^{\mathrm{a}, \dagger}$

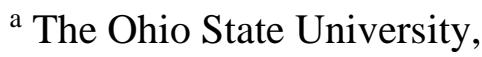 \\ Department of Materials Science and Engineering, \\ Edison Joining Technology Center, \\ 1248 Arthur E. Adams Dr. Columbus, OH 43221, United States
}

${ }^{\mathrm{b}}$ Imperial College London,

Department of Mechanical Engineering,

Exhibition Road, London SW7 2AZ, United Kingdom

\footnotetext{
${ }^{\dagger}$ Electronic mail: rokhlin.2@osu.edu

Manuscript accepted for publication in J. Acoust. Soc. Am. 147 (4), April 2020.
} 


\section{Abstract}

2 Better understanding of elastic wave propagation in polycrystals has interest for applications

3 in seismology and nondestructive material characterization. In this study, a second-order

4 wave propagation (SOA) model that considers forward multiple scattering events is

5 developed for macroscopically isotropic polycrystals with equiaxed grains of arbitrary

6 anisotropy (triclinic). It predicts scattering-induced wave attenuation and dispersion of phase

7 velocity. The SOA model implements the generalized two-point correlation (TPC) function,

8 which relates to the actual numeric TPC of simulated microstructure. The analytical Rayleigh

9 and stochastic asymptotes for both attenuation and phase velocity are derived for triclinic

10 symmetry grains, which elucidate the effects of the elastic scattering factors and the

11 generalized TPC in different frequency regimes. Also, the computationally efficient far field

12 approximation (FFA) attenuation model is obtained for this case; it shows good agreement

13 with the SOA model in all frequency ranges. To assess the analytical models, a 3D finite

14 element (FE) model for triclinic polycrystals is developed and implemented on simulated 3D

15 triclinic polycrystalline aggregates. Quantitative agreement is observed between the

16 analytical and the FE simulations for both the attenuation and phase velocity. Also, the quasi-

17 static velocities obtained from the SOA and FE models are in excellent agreement with the

18 static self-consistent velocity.

19 Keywords: wave scattering, general anisotropy grains, analytical model, finite element

20 method, attenuation and velocity, two-point correlation function 


\section{INTRODUCTION}

23 Most nature- and man-made solid materials are inherently inhomogeneous. When an elastic wave propagates in a randomly inhomogeneous medium, for example a polycrystal, it scatters energy from inhomogeneities into longitudinal and transverse waves. The resulting incoherent scattering leads to the attenuation and dispersion of the main coherent propagating wave. The incoherent scattering energy eventually decays due to absorption mechanisms in the solid. The absorption attenuation is usually weak in comparison to the scattering-induced attenuation, so the study of the attenuation of the coherent propagating wave in polycrystals can usually neglect consideration of the absorption mechanisms. Theoretical, numerical and experimental developments in this active field are of great interest due to numerous practical applications in seismology and nondestructive testing [1-3].

Metal and ceramic polycrystals are widely used in industry and our everyday life. Modeling [4-10] of ultrasonic wave scattering in such materials not only improves our understanding of wave interaction phenomena but is also important for signal interpretation and signal-to-noise ratio improvement during ultrasonic nondestructive evaluation [3] of those materials. Also, one can potentially obtain information about material microstructure using model-based inversion of ultrasonic experimental scattering data [9]. Realistic microstructures of polycrystalline materials, especially engineering alloys, are very complex and may include multiple phases, columnar grains and crystallographic texture. Driven by applications of ultrasonic characterization of industrial polycrystalline alloys, analytical wave scattering models have been developed for polycrystals with low symmetry crystallites $[8,11-13]$, ellipsoidal grain shapes $[8,9,13,14,15]$, crystallographic texture $[7,8,12,16]$ and residual stresses [17]. Also, complex colony microstructure of titanium alloys [18] can be incorporated in the scattering models through the effective grain properties of general anisotropy $[8,12]$. 
47 The above recent advances in analytical modeling of wave propagation in polycrystals are based on the original Stanke and Kino [4] and Weaver [5] models, which result in equivalent dispersion equations for the perturbed wave number. In the current work, following [4], we call these models second order approximation (SOA) models. In these scattering models the actual polycrystalline structure, with the elastic tensor abruptly changing through the grain boundaries from crystallite to crystallite, is replaced by a continuous elastic medium with random fluctuations of the elastic tensor with statistical properties approximating those of polycrystalline media. Such a replacement avoids solution of a dynamic boundary value problem. The wave propagation in this replaced-material model is solved by a perturbative approximate analytic method. The level of error of those approximations is generally unknown; however, it is understood that the error decreases with decrease of material inhomogeneity perturbation (which depends on crystallite anisotropy). In most modeling efforts mentioned above, except for $[4,13,15,19]$, further approximations are employed by invoking the Born approximation. The limitations of the Born approximation are that it breaks in transition from the stochastic to the geometric region $[4,13,19,20]$ and that it has larger discrepancy with the Stanke and Kino model[4] for cubic polycrystals with a large Zener anisotropy factor $[4,13]$.

Therefore, there are important fundamental and practical needs in establishing the range of validity of those models in predicting ultrasonic phase velocity and attenuation for a variety of material systems characterized by different levels of the perturbation parameter and grain crystallographic symmetries. These needs may be addressed by 3D numerical modeling of scattering in the representative material systems. Recent progress in 3-D numerical modeling of elastic wave scattering in polycrystals [21-26] has opened new opportunities to study the effect of microstructure on wave propagation phenomena in heterogeneous media and elucidate the applicability of the above-mentioned analytical models. Those numerical 
methods of elastic wave scattering employ material system representation, usually by 3D

73 Voronoi tessellation grain models, their finite element (FE) discretization and dynamic wave scattering simulations. FE computations account for full-wave physics models with control and knowledge of the random properties of the materials and incorporate the complete chain of multiscattering events. In validation of analytical models the finite element method has several advantages over experiment: control [23] of the inhomogeneity perturbation parameters $[4,20]$ of the simulated polycrystal; creating the required Voronoi grain microstructures by specially-designed algorithms [27]; accurate determination of the twopoint and other statistics of the simulated 3D polycrystalline systems and employing fitted statistics in the analytical models. Those features are difficult to achieve and/or to measure for material samples used for experimental ultrasonic measurements of the attenuation, velocity or scattering in polycrystals.

In our prior work [21,23] we have evaluated second-order analytical models (of the type of Stanke and Kino [4]) by comparison with the 3D FE simulations for material systems with cubic crystallographic grain symmetries. A range of polycrystals, from $\mathrm{Al}$ to $\mathrm{Cu}$ have been studied with Zener anisotropic factors $1.24 \leq A \leq 3.14$ ( $\mathrm{A}=1$ is isotropy). The models were developed to include the numerically obtained two-point correlation functions (TPC) on the simulated 3D Voronoi grain microstructures of polycrystals; those TPCs were accurately fitted by exponential series $[21,23]$ for implementation in the analytical models (we term such fitted correlation functions generated TPC functions). The results showed good agreement between the analytical and the FE models (FEMs) indicating proper accounting for effects of inhomogeneity and multiple scattering by the analytical model even for polycrystals with high anisotropy grains such as $\mathrm{Ni}$ and $\mathrm{Cu}$.

No development of the second order approximation (SOA) analytical models or the 3D FEM has been done in the past for polycrystals with crystallographic symmetry of grains lower 
than cubic. However, practically-important Ti alloys have hexagonal or even lower symmetry of grains. For instance, the $\alpha / \beta$ colonies that exist in aerospace titanium alloys forming

99 effective grains of monoclinic symmetry [18], Also, some steels with martensite and bainite microstructures have low symmetry grains.

101 To fill this gap, we seek to formulate and implement the second-order approximation (SOA) analytical models and the 3D FE model for elastic wave propagation in polycrystals with equiaxed grains of general (triclinic) crystallographic symmetry (having 21 independent elastic constants). The analytical models developed incorporate the generalized TPC, obtained from the simulated FEM material systems, and are applicable in all frequency ranges as the Stanke-Kino model [4]. The explicit equations for the wavenumber asymptotes in the Rayleigh and stochastic regimes are also derived; previously they were known for cubic materials $[4,13]$ only. Next, we meet the challenge of the development of the 3D FEM material models with triclinic crystallites and the implementation of the 3D FE model to

110 determine the attenuation and velocity over a large frequency range; the FE approach for 111 obtaining the quasi-static velocity limit is also implemented.

112 Wave scattering in an aggregate of triclinic crystallites is affected by all 21 elastic constants 113 of triclinic grains, which complicates the wave-scattering analysis modelling and physical 114 interpretation. We were able to show that the crystallite elastic constants mostly act through 115 their combination expressed by the elastic scattering factors that appear in the Rayleigh and stochastic asymptotes and the far-field approximation (FFA) model [13]. These coefficients

117 play a major role in the data interpretation when we are comparing the analytical and the FE

118 model results for all material systems studied in this and the prior [21,23] work.

119 In Sec. II, we present the second-order analytical attenuation and velocity models with the 120 generalized two-point correlation function for triclinic polycrystals, considering the SOA and 
121 FFA models. Sec. III addresses the FE methodology for wave scattering in triclinic 122 polycrystalline materials. Quantitative comparison between analytical models and 3D FEM

123 for attenuation and phase velocity is addressed in Sec. IV, where we also briefly discuss the 124 implication of our results for the backscattering coefficient. Finally, the conclusions are 125 given. MODELS WITH GENERALIZED TPC FOR POLICRYSTALS WITH TRICLINIC GRAINS

In this paper, we consider propagation and scattering of elastic waves in fully dense polycrystalline media with equiaxed grains of general anisotropy (triclinic). The grains are randomly oriented and continuity of stresses and displacements is assumed on the grain boundaries. The propagating wave scatters on grain boundaries due to grain stiffness misorientations leading to wave attenuation and dispersion. These are called grain-scatteringinduced attenuation and dispersion that must be distinguished from the wave absorption and dispersion on imperfections of a crystalline structure which are neglected in this work. To address this wave interaction problem one needs to solve a $3 \mathrm{D}$ dynamic boundary value

137 problem of multiple scattering on all grains. Currently, the exact theoretical solution is

138 beyond the apparent limits of theoretical models. For this reason, as discussed in the 139 introduction, approximate analytical models, based on the approach of references [4,5], have 140 been developed.

141 The fundamental analytical models of elastic wave scattering in a polycrystal [4,5] first start with the replacement of the polycrystal by a random continuous medium with a small random

143 spatial fluctuation $\delta c_{i j k l}(\mathbf{X})$ of the elastic tensor $c_{i j k l}(\mathbf{X})$ around its mean $\left\langle c_{i j k l}\right\rangle, \mathbf{X}$ is a

144 coordinate point in a polycrystal. The grain statistics (size and orientation) of the polycrystal 
145 is represented in the model by the two-point correlation function (TPC) $\left\langle\delta c_{i j k l}(\mathbf{X}) \delta c_{\alpha \beta \gamma \delta}\left(\mathbf{X}^{\prime}\right)\right\rangle$

146 for the fluctuation $\delta c_{i j k l}(\mathbf{X})$. The TPC describes the probability of two points separated by a

147 distance $r$ falling into the same grain; it therefore characterizes the microstructure geometry

148 (such as size, shape of grains) [4,5,12,20]. It can be factorized [4,5] as

$149\left\langle\delta c_{i j k l}(\mathbf{X}) \delta c_{\alpha \beta \gamma \delta}\left(\mathbf{X}^{\prime}\right)\right\rangle=\left\langle\delta c_{i j k l} \delta c_{\alpha \beta \gamma \delta}\right\rangle w(r)$, where the first factor is the elastic covariance and

150 the second, $w(r)$, is the geometrical TPC with $r=\left|\mathbf{X}-\mathbf{X}^{\prime}\right|$. For brevity, from here on in the

151 paper, will refer to the geometrical TPC function $w(r)$ as simply TPC.

152 In this section, we first describe the generalized TPC used in this paper for the analytical

153 models and their comparison with the FEM. It follows by a description of the second order

154 approximation (SOA) model applicable in all frequency ranges, as the Stanke and Kino

155 model [4], but for polycrystals with grains of general anisotropy (triclinic) and the

156 generalized TPC. Next, in this general case we derive the Rayleigh and stochastic asymptotes

157 for the perturbed wave number, thus providing the asymptotes not only for the attenuation but

158 also for the phase velocity. Such wave number asymptotes were known in the past for cubic

159 polycrystals only [4, 13]. Finally, the far field approximation (FFA) model is briefly given for

160 further comparison with the SOA and the FEM results.

\section{A. Generalized TPC function}

162 In most prior theoretical work on wave propagation in polycrystals the exponential form of

163 the TPC $\exp (-r / a)$ ( $a$ is the mean grain radius) has been adopted for polycrystals with

164 equiaxed [4,5,7,13,19,28-32] and ellipsoidal grains [9,12-15,29,30]. However, it was shown

165 experimentally [30] that a polycrystal TPC may deviate significantly from a simple

166 exponential form. Recent 3D finite element simulation studies [21-23] of elastic wave

167 propagation in cubic polycrystals with numerically created polycrystalline microstructures 
also showed a numerically determined TPC of the simulated microstructures that deviated

169 from exponential form. Comparison of the analytical attenuation models with the 3D FEM

170 showed that the results are in good agreement when the actual fitted TPC functions of

171 polycrystals are used in the analytical models.

172 In our prior work [21,23], we have introduced for cubic polycrystals with equiaxed grains the 173 generalized TPC represented by an exponential series:

$174 \quad w(r)=\sum_{i=1}^{N} A_{i} \exp \left(-\frac{r}{a_{i}}\right)$

175 where $A_{i}$ and $a_{i}\left(a_{i}>0\right)$ are constants, which are determined by fitting of the actual TPC 176 obtained numerically for the simulated FE microstructure. The TPC function, due to the 177 assumption of macroscopic isotropy and equiaxed shape of the grains, has no directional 178 dependence and depends solely on the distance $r$. In this work we are considering 179 polycrystals formed by triclinic equiaxed grains. Because of the fundamental importance of 180 the generalized TPC for this work, we briefly outline its main properties below.

181 Physically, the probability for two points to be in the same grain when $r=0$ should be unity,

182 therefore we obtain a constraint on the coefficients $A_{i}$ :

$183 \quad \sum_{\mathrm{i}=1}^{N} A_{i}=1$

184 As it is mathematically proved in Ref. [30], the derivative of the TPC function in the vicinity 185 of the origin $r=0$ is related to the mean line intercept determined from the microstructure 186 cross-sections; this gives the second constraint on the coefficients in the Eq. (1) series:

$187 \quad w^{\prime}(r=0)=\left.\frac{d w(r)}{d r}\right|_{r=0}=-\sum_{i=1}^{N} \frac{A_{i}}{a_{i}}=-\frac{1}{a_{l}}$, 
188 where prime donates derivative with respect to $\mathrm{r}$, and $a_{l}$ is the mean linear intercept (chord 189 length). For an exponential TPC function, the two conditions Eqs. (2) and (3), are satisfied 190 automatically; for a generalized TPC, the coefficients $A_{i}$ and $a_{i}$ are determined by nonlinear191 least-square fitting of the measured TPC data (the fitting is discussed in Sec. III). As has been 192 discussed in reference [23], we do not attribute a physical meaning to each exponential term 193 in Eq. (1) and we apply general physical requirements, Eqs. (2,3), of the two-point correlation 194 function only to the total sum of Eq. (1). We discuss the fitting of the actual material TPC 195 function by the series (1) in Sec. III.C.2.

196 Another important property of the random medium is the correlation length $a_{C L}$. Usually it is

197 defined as [33] $a_{C L}=\int_{0}^{\infty} w(r) d r$, and therefore for the generalized TPC the correlation 198 length is determined as $a_{C L}=\sum_{j=1}^{N} A_{j} a_{j}$. This is consistent with the definition of the correlation 199 length [32] for the polycrystal with the exponential TPC, $\exp (-r / a)$, as $a_{C L}=a$. In this last 200 case $a$ is also equal to the mean line intercept and is associated with the mean grain radius 201 [32]. It is also useful to consider an effective grain volume $V_{g}^{\text {eff }}=\int_{V} w(r) d V$ that was 202 discussed by Stanke [32]. This characteristic plays an important role in the Rayleigh 203 scattering regime. Integrating Eq. (1) over a polycrystal volume we have $V_{g}^{e f f}=8 \pi \sum_{j=1}^{N} A_{j} a_{j}^{3}$.

204 For the exponential TPC, $\exp (-r / a)$, we immediately obtain $V_{g}^{\text {eff }}=8 \pi a^{3}$ [32].

205 Representation of the actual TPC of a random medium by the exponential series Eq. (1) has 206 the advantage of a simpler theoretical formulation, especially for the FFA and Born 207 approximations and the Rayleigh and stochastic asymptotes (see ref [23] and later in this 208 work). 
B. The second order scattering model for perturbed wave number in polycrystals with equiaxed grains of general anisotropy

\section{1. Second order approximation (SOA) scattering model}

212 The second order attenuation model for longitudinal wave scattering based on the Dyson

213 equation [5] has been developed for cubic polycrystalline materials with generalized TPC,

214 Eq. (1), in Ref. [23]. Following the same procedure, the SOA model is extended here for the

215 longitudinal and transverse wave propagation in polycrystals with equiaxed triclinic grains.

216 Using the notations established in Refs. [13, 23], the dispersion equations of the SOA model

217 with generalized TPC function both for longitudinal (L) and transverse (T) incident waves

218 can be written in a compact form:

$219 k_{0 M}^{2}-k^{2}-m_{M}(\mathbf{k}) / V_{0 M}^{2}=0$

where $\mathbf{k}=k \mathbf{p}$ is a perturbed complex wavenumber in the wave propagation direction defined

by a unit vector $\mathbf{p}$, subscript $M$ identifies the wave mode of an incident wave ( $\mathrm{L}$ or $\mathrm{T})$,

$m_{M}(\mathbf{k})$ is the mass operator or the self-energy operator[5], and $k_{0 M}, V_{0 M}$ are the wavenumber

and the Voigt velocity respectively for a homogeneous reference medium[23] (the Voigt

elastic constants and velocity for a homogenized triclinic polycrystal are given in Appendix

A). As a result of the mode conversion, the mass operator for the incident wave $M$ ( $\mathrm{L}$ or T) is a summation of two terms representing scattering into $\mathrm{L}, \mathrm{T}$ waves, namely $m_{M}(\mathbf{k})=m_{M \rightarrow L}(\mathbf{k})+m_{M \rightarrow T}(\mathbf{k})$. Note that due to macroscopic isotropy the two shear waves,

SH and SV, are identical and therefore only two terms of the mass operator are considered; for textured polycrystals with macroscopic anisotropy three scattering components of the mass operator should be considered $[7,8,13]$.

231 For longitudinal and transverse wave propagation and the generalized TPC, the individual

232 components (terms) for the mass operator, following notations of Ref [23], are written as: 
$m_{L \rightarrow L}(k)=\frac{k^{2} k_{0 L}^{3}}{\rho^{2} V_{0 L}^{2}}\left\{\frac{2}{\pi} P . V \cdot \int_{0}^{\infty} \frac{\xi^{4}}{1-\xi^{2}} \int_{-1}^{+1}\left[\sum_{j=1}^{N} A_{j} a_{j}^{3} f_{L L}\left(k, a_{j}, x, \xi\right)\right] d x d \xi-i \int_{-1}^{+1}\left[\sum_{j=1}^{N} A_{j} a_{j}^{3} f_{L L}\left(k, a_{j}, x, \xi=1\right)\right] d x\right\}$

234

$m_{L \rightarrow T}(k)=\frac{k^{2} k_{0 T}^{3}}{\rho^{2} V_{0 T}^{2}}\left\{\frac{2}{\pi} P . V \cdot \int_{0}^{\infty} \frac{\xi^{4}}{1-\xi^{2}} \int_{-1}^{+1}\left[\sum_{j=1}^{N} A_{j} a_{j}^{3} f_{L T}\left(k, a_{j}, x, \xi\right)\right] d x d \xi-i \int_{-1}^{+1}\left[\sum_{j=1}^{N} A_{j} a_{j}^{3} f_{L T}\left(k, a_{j}, x, \xi=1\right)\right] d x\right\}$

235

$$
m_{T \rightarrow L}(k)=\frac{k^{2} k_{0 L}^{3}}{2 \rho^{2} V_{0 L}^{2}}\left\{\frac{2}{\pi} P . V \cdot \int_{0}^{\infty} \frac{\xi^{4}}{1-\xi^{2}} \int_{-1}^{+1}\left[\sum_{j=1}^{N} A_{j} a_{j}^{3} f_{T L}\left(k, a_{j}, x, \xi\right)\right] d x d \xi-i \int_{-1}^{+1}\left[\sum_{j=1}^{N} A_{j} a_{j}^{3} f_{T L}\left(k, a_{j}, x, \xi=1\right)\right] d x\right\}
$$

$$
m_{T \rightarrow T}(k)=\frac{k^{2} k_{0 T}^{3}}{2 \rho^{2} V_{0 T}^{2}}\left\{\frac{2}{\pi} P \cdot V \cdot \int_{0}^{\infty} \frac{\xi^{4}}{1-\xi^{2}} \int_{-1}^{+1}\left[\sum_{j=1}^{N} A_{j} a_{j}^{3} f_{T T}\left(k, a_{j}, x, \xi\right)\right] d x d \xi-i \int_{-1}^{+1}\left[\sum_{j=1}^{N} A_{j} a_{j}^{3} f_{T T}\left(k, a_{j}, x, \xi=1\right)\right] d x\right\}
$$

237 Where $i$ before the second terms is the imaginary unit, P.V. means the Cauchy principal 238 value, $\xi$ is a non-dimensional integration variable, $\rho$ is material density, and the complex 239 functions $f_{L L}, f_{L T}, f_{T L}, f_{T T}$ are defined as:

240

$$
f_{L L}\left(k, a_{j}, x, \xi\right)=\frac{A_{L L}+B_{L L} x^{2}+C_{L L} x^{4}}{\left[\left(1+k^{2} a_{j}^{2}+\xi^{2} k_{0 L}^{2} a_{j}^{2}\right)-2 k \xi k_{0 L} a_{j}^{2} x\right]^{2}}
$$

$$
f_{L T}\left(k, a_{j}, x, \xi\right)=\frac{A_{L T}+B_{L T} x^{2}+C_{L T} x^{4}}{\left[\left(1+k^{2} a_{j}^{2}+\xi^{2} k_{0 T}^{2} a_{j}^{2}\right)-2 k \xi k_{0 T} a_{j}^{2} x\right]^{2}}
$$

242

$$
f_{T L}\left(k, a_{j}, x, \xi\right)=\frac{A_{L T}+B_{L T} x^{2}+C_{L T} x^{4}}{\left[\left(1+k^{2} a_{j}^{2}+\xi^{2} k_{0 L}^{2} a_{j}^{2}\right)-2 k \xi k_{0 L} a_{j}^{2} x\right]^{2}}
$$

$243 f_{T T}\left(k, a_{j}, x, \xi\right)=\frac{A_{T T}+B_{T T} x^{2}+C_{T T} x^{4}}{\left[\left(1+k^{2} a_{j}^{2}+\xi^{2} k_{0 T}^{2} a_{j}^{2}\right)-2 k \xi k_{0 T} a_{j}^{2} x\right]^{2}}$

244 Those functions are complex since the perturbed wave number is complex. The related 245 functions $f_{N M}$ in the single integrals of the second terms in Eqs. (5-8) are obtained after 246 substituting $\xi=1$ into the functions (9-12). 
247 In the equations above, the inner product coefficients $A_{L L}, B_{L L}, C_{L L}, A_{L T}, B_{L T}, C_{L T}$, $248 A_{T T}, B_{T T}, C_{T T}$ and Voigt velocities $V_{0 L}, V_{0 T}$ for texture-free polycrystals with triclinic 249 symmetry grains are specified in Appendix A. The generalized TPC function (1) is 250 incorporated into the SOA model; the SOA model with statistics represented by a single 251 exponent TPC is obtained by choosing $N=1$. The integrals in Eqs. (5-8) and the dispersion 252 equation (4) are solved numerically.

\section{2. The Born approximation}

254 Significant simplifications of the SOA model are obtained by application of the Born 255 approximation. It allows us to derive the analytical Rayleigh and stochastic asymptotes for 256 the phase velocity and attenuation. The Born approximation is obtained from the dispersion 257 equation (4) by replacing the perturbed wave number $\mathrm{k}$ in the mass operator $m_{M}(k)$ by that 258 of the reference medium, $k_{0 M}$. Due to the small perturbation of the wavenumber $259\left(\operatorname{Re} k-k_{0 L}\right)<<1$, we also substitute, as in $\operatorname{Ref[4],} k^{2}-k_{0 L}^{2} \approx 2 k_{0 L}\left(k-k_{0 L}\right)$ and obtain an 260 explicit equation for the perturbed wave number:

$k=k_{0 L}-\frac{m_{L}\left(k_{0 L}\right)}{2 k_{0 L} V_{0 L}^{2}}, \quad m_{L}\left(k_{0 L}\right)=m_{L \rightarrow L}\left(k_{0 L}\right)+m_{L \rightarrow T}\left(k_{0 L}\right)$.

262 In this form of the Born approximation the Cauchy integrals still remain in the mass operators. It is important to note that after replacement in the mass operators of the perturbed

264 complex wave number $k$ by the real value $k_{0 L}$ the functions (9-12) become real. Therefore 265 in Eqs. (5-8) the real and imaginary parts of the mass operator are automatically separated;

266 with the real part being the first term with the double integral, and the imaginary part the

267 second term with the single integral. This is significant since the attenuation coefficient is 
obtained from the imaginary part of the perturbed wave number and the wave phase velocity

269 from the real part.

270 Next, we discuss below the Rayleigh and stochastic asymptotes for the wave phase velocity 271 and attenuation (the integrals are evaluated analytically for those asymptotes in Appendices B 272 and C).

273 C. The Rayleigh asymptotes for attenuation and quasi-static velocity limits 274 from the SOA model

275 Here we consider the longitudinal and transverse wave attenuation and phase velocity in 276 triclinic polycrystals with equiaxed grains at low frequency (Rayleigh scattering regime). In

277 this regime the wavelength is much larger than the scatterer sizes $\lambda>>a_{i}\left(k_{0 L} a_{i}, k_{0 T} a_{i}<<1\right)$.

278 We consider this approximation for the perturbed wave number thus obtaining the asymptotes 279 for both the quasi-static velocity limits and attenuation asymptotes for the general anisotropy 280 grains (triclinic) and generalized TPC Eq. (1). This provides better understanding of the scattering phenomenon at a long wavelength. The obtained novel quasi-static velocity limits have special importance as discussed in Sec. IV.C.

283 As discussed in Appendix B, we have derived from the Born approximation for the low 284 frequency the explicit results of the Cauchy principal values in the mass operators by 285 complex integration and the residue theorem. As a result, the Rayleigh asymptote for the 286 normalized longitudinal perturbed complex wavenumber $k \rightarrow k_{L}^{R}$ is:

287

$$
\begin{aligned}
& \frac{k_{L}^{R}}{k_{0 L}}=1+\frac{\left(A_{L L}+B_{L L} / 3+C_{L L} / 5\right)}{\left\langle c_{11}\right\rangle^{2}}\left(\frac{1}{2}+i k_{0 L}^{3} \sum_{j=1}^{N} A_{j} a_{j}^{3}\right)+\frac{\left(A_{L T}+B_{L T} / 3+C_{L T} / 5\right)}{\left\langle c_{11}\right\rangle\left\langle c_{44}\right\rangle}\left(\frac{1}{2}+i k_{0 T}^{3} \sum_{j=1}^{N} A_{j} a_{j}^{3}\right) \\
& =1+4 Q_{L L}^{*}\left(\frac{1}{2}+i k_{0 L}^{3} \sum_{j=1}^{N} A_{j} a_{j}^{3}\right)+4 Q_{L \rightarrow T}\left(\frac{1}{2}+i k_{0 T}^{3} \sum_{j=1}^{N} A_{j} a_{j}^{3}\right)
\end{aligned}
$$


where $\left\langle c_{11}\right\rangle$ and $\left\langle c_{44}\right\rangle$ are the elastic constants of the Voigt reference medium, and the sum $8 \pi \sum_{j=1}^{N} A_{j} a_{j}^{3}=V_{g}^{e f f}$ is defined as the effective grain volume (Sec. II.A). Scattering factors Q in

290 Eq. (9) are defined as $Q_{L L}^{*}=\frac{\left(A_{L L}+B_{L L} / 3+C_{L L} / 5\right)}{4\left\langle c_{11}\right\rangle^{2}}, Q_{L \rightarrow T}=\frac{\left(A_{L T}+B_{L T} / 3+C_{L T} / 5\right)}{4\left\langle c_{11}\right\rangle\left\langle c_{44}\right\rangle}\left(Q_{L \rightarrow T}\right.$

291 follows the same definition as in Ref.[13], the factor $Q_{L L}^{*}$ is introduced to simplify notation).

292 The quasi-static phase velocity is obtained from the real part of the perturbed wave number 293 while the attenuation is from the imaginary part:

294

$$
\begin{aligned}
& V_{L}^{R}=V_{L}^{q-s t}=V_{0 L}\left[1+\frac{1}{2\left\langle c_{11}\right\rangle^{2}}\left(A_{L L}+B_{L L} / 3+C_{L L} / 5\right)+\frac{1}{2\left\langle c_{11}\right\rangle\left\langle c_{44}\right\rangle}\left(A_{L T}+B_{L T} / 3+C_{L T} / 5\right)\right]^{-1} \\
& =V_{0 L}\left[1+2 Q_{L L}^{*}+2 Q_{L \rightarrow T}\right]^{-1}
\end{aligned}
$$

295

$$
\begin{aligned}
& \alpha_{L}^{R}=\frac{k_{0 L}^{4}}{\left\langle c_{11}\right\rangle^{2}}\left(A_{L L}+B_{L L} / 3+C_{L L} / 5\right) \sum_{j=1}^{N} A_{j} a_{j}^{3}+\frac{k_{0 T}^{3} k_{0 L}}{\left\langle c_{11}\right\rangle\left\langle c_{44}\right\rangle}\left(A_{L T}+B_{L T} / 3+C_{L T} / 5\right) \sum_{j=1}^{N} A_{j} a_{j}^{3} \\
& =4 k_{0 L}^{4} Q_{L L}^{*} \sum_{j=1}^{N} A_{j} a_{j}^{3}+4 k_{0 T}^{3} k_{0 L} Q_{L \rightarrow T} \sum_{j=1}^{N} A_{j} a_{j}^{3}
\end{aligned}
$$

296 The scattering factors $Q_{L L}^{*}$ and $Q_{L \rightarrow T}$ are given analytically for the triclinic symmetry in

297 Appendix D; they are represented in terms of a sum of quadratic terms of elastic constants.

298 Similarly, the Rayleigh limit of the normalized complex wavenumber for a transverse 299 incident wave is:

$$
\begin{aligned}
& \frac{k_{T}^{R}}{k_{0 T}}=1+\frac{\left(A_{T T}+B_{T T} / 3+C_{T T} / 5\right)}{2\left\langle c_{44}\right\rangle^{2}}\left(\frac{1}{2}+i k_{0 T}^{3} \sum_{j=1}^{N} A_{j} a_{j}^{3}\right)+\frac{\left(A_{L T}+B_{L T} / 3+C_{L T} / 5\right)}{2\left\langle c_{11}\right\rangle\left\langle c_{44}\right\rangle}\left(\frac{1}{2}+i k_{0 L}^{3} \sum_{j=1}^{N} A_{j} a_{j}^{3}\right) \\
& =1+4 Q_{T T}^{*}\left(\frac{1}{2}+i k_{0 T}^{3} \sum_{j=1}^{N} A_{j} a_{j}^{3}\right)+4 Q_{T \rightarrow L}\left(\frac{1}{2}+i k_{0 L}^{3} \sum_{j=1}^{N} A_{j} a_{j}^{3}\right)
\end{aligned}
$$


301 Where the two scattering factors $Q_{T T}^{*}$ and $Q_{T \rightarrow L}$ in Eq. (17) are defined as

$Q_{T T}^{*}=\frac{\left(A_{T T}+B_{T T} / 3+C_{T T} / 5\right)}{8\left\langle c_{44}\right\rangle^{2}}, Q_{T \rightarrow L}=\frac{\left(A_{L T}+B_{L T} / 3+C_{L T} / 5\right)}{8\left\langle c_{11}\right\rangle\left\langle c_{44}\right\rangle}$ (this follows the same definition as in

303 Ref. [13]). From the equation above, the Rayleigh limit of the phase velocity (quasi-static

305

$$
\begin{aligned}
& V_{T}^{R}=V_{T}^{q-s t}=V_{0 T}\left[1+\frac{\left(A_{T T}+B_{T T} / 3+C_{T T} / 5\right)}{4\left\langle c_{44}\right\rangle^{2}}+\frac{\left(A_{L T}+B_{L T} / 3+C_{L T} / 5\right)}{4\left\langle c_{11}\right\rangle\left\langle c_{44}\right\rangle}\right]^{-1} \\
& =V_{0 T}\left[1+2 Q_{T T}^{*}+2 Q_{T \rightarrow L}\right]^{-1}
\end{aligned}
$$

306

$$
\alpha_{T}^{R}=\frac{k_{0 T}^{4}\left(A_{T T}+B_{T T} / 3+C_{T T} / 5\right)}{2\left\langle c_{44}\right\rangle^{2}} \sum_{j=1}^{N} A_{j} a_{j}^{3}+\frac{k_{0 L}^{3} k_{0 T}\left(A_{L T}+B_{L T} / 3+C_{L T} / 5\right)}{2\left\langle c_{44}\right\rangle\left\langle c_{11}\right\rangle} \sum_{j=1}^{N} A_{j} a_{j}^{3}
$$

$=4 k_{0 T}^{4} Q_{T T}^{*} \sum_{j=1}^{N} A_{j} a_{j}^{3}+4 k_{0 L}^{3} k_{0 T} Q_{T \rightarrow L} \sum_{j=1}^{N} A_{j} a_{j}^{3}$

307 The scattering factor $Q_{T \rightarrow L}=Q_{L \rightarrow T} / 2$ is obtained from Appendix D; and the factor $Q_{T T}^{*}$ is

308 obtained from the analytical form of the coefficients $A_{T T}, B_{T T}, C_{T T}$ given in ref. [44].

309 The explicit equations obtained in this section for the Rayleigh asymptotes show that the

310 quasi-static velocities for the $\mathrm{L}$ and $\mathrm{T}$ waves have no dependence on the TPC function and

311 therefore they are independent of the grain size and depend only on the elastic covariances of

312 the grains. However the Rayleigh asymptotes for attenuation coefficients are strongly

313 dependent on the TPC and are proportional to the effective grain volume $V_{g}^{\text {eff }}$.

\section{D. The stochastic asymptotes for attenuation and velocity}

315 The stochastic asymptotes for the perturbed wave number are obtained directly from the SOA

316 model after applying the Born approximation Eq. (13). In the stochastic regime (high

317 frequency) $k_{0 L} a_{i}>>1$ the Cauchy principal integral can be obtained analytically (appendix

318 C). The stochastic asymptote of the normalized longitudinal perturbed wavenumbers is: 


$$
\begin{aligned}
& \frac{k_{L}^{S}}{k_{0 L}}=1-\frac{\left(A_{L T}+B_{L T}+C_{L T}\right)}{2\left\langle c_{11}\right\rangle\left(\left\langle c_{11}\right\rangle-\left\langle c_{44}\right\rangle\right)}+\frac{\left(A_{L L}+B_{L L}+C_{L L}\right)}{4\left\langle c_{11}\right\rangle^{2}}\left(\frac{5}{2}+i k_{0 L} \sum_{j=1}^{N} A_{j} a_{j}\right) \\
& =1-\frac{\left(A_{L T}+B_{L T}+C_{L T}\right)}{2\left\langle c_{11}\right\rangle\left(\left\langle c_{11}\right\rangle-\left\langle c_{44}\right\rangle\right)}+Q_{L \rightarrow L}\left(\frac{5}{2}+i k_{0 L} \sum_{j=1}^{N} A_{j} a_{j}\right)
\end{aligned}
$$

320 where $\sum_{j=1}^{N} A_{j} a_{j}$ is the correlation length $a_{C L}$ for the generalized TPC function (Sec. II.A),

$321 a_{C L}=\int_{0}^{\infty} w(r) d r=\sum_{j=1}^{N} A_{j} a_{j}$. One should note that under the exponential TPC assumption the

322 free path in the stochastic regime is $2 a_{l}$, Ref. [32]; however, in this study the free path is

323 different given the use of the generalized TPC function. The scattering factor $Q_{L \rightarrow L}$ in Eq.

324 (20) follows the definition of Ref. [13] for triclinic crystallites; it relates to the inhomogeneity 325 parameter discussed in Ref. [4,20].

326 It is important that the real part of the perturbed wavenumber in Eq. (20) depends on the 327 longitudinal wave scattering into both the longitudinal (LL coefficients) and transverse (LT 328 coefficients) waves. The imaginary part accounts for scattering into the longitudinal wave 329 only; this is in line with the well-known fact that in the stochastic regime for the attenuation, 330 the scattering into the transverse wave can be neglected.

331 From Eq. (20), the asymptotes for longitudinal phase velocity and attenuation in the high 332 frequency limit are:

333

$$
\begin{aligned}
& V_{L}^{S}=V_{0 L}\left[1+\frac{5\left(A_{L L}+B_{L L}+C_{L L}\right)}{8\left\langle c_{11}\right\rangle^{2}}-\frac{\left(A_{L T}+B_{L T}+C_{L T}\right)}{2\left\langle c_{11}\right\rangle\left(\left\langle c_{11}\right\rangle-\left\langle c_{44}\right\rangle\right)}\right]^{-1} \\
& =V_{0 L}\left[1+\frac{5 Q_{L \rightarrow L}}{2}-\frac{\left(A_{L T}+B_{L T}+C_{L T}\right)}{2\left\langle c_{11}\right\rangle\left(\left\langle c_{11}\right\rangle-\left\langle c_{44}\right\rangle\right)}\right]^{-1}
\end{aligned}
$$

$$
\alpha_{L}^{S}=\frac{k_{0 L}^{2}\left(A_{L L}+B_{L L}+C_{L L}\right)}{4\left\langle c_{11}\right\rangle^{2}} \sum_{j=1}^{N} A_{j} a_{j}=k_{0 L}^{2} Q_{L \rightarrow L} \sum_{j=1}^{N} A_{j} a_{j}
$$


335 The scattering factor $Q_{L \rightarrow L}$ is given analytically for triclinic symmetry in Appendix A, and

336 the factor $\Delta_{9}=\left(A_{L T}+B_{L T}+C_{L T}\right)$ is given in Appendix D.2.

337 Similarly, the stochastic asymptote for the normalized transverse perturbed wavenumber is 338 obtained explicitly:

339

$$
\begin{aligned}
& \frac{k_{T}^{S}}{k_{0 T}}=1+\frac{\left(A_{L T}+B_{L T}+C_{L T}\right)}{4\left\langle c_{44}\right\rangle\left(\left\langle c_{11}\right\rangle-\left\langle c_{44}\right\rangle\right)}+\frac{\left(A_{T T}+B_{T T}+C_{T T}\right)}{8\left\langle c_{44}\right\rangle^{2}}\left(\frac{5}{2}+i k_{0 T} \sum_{j=1}^{N} A_{j} a_{j}\right) \\
& =1+\frac{\left(A_{L T}+B_{L T}+C_{L T}\right)}{4\left\langle c_{44}\right\rangle\left(\left\langle c_{11}\right\rangle-\left\langle c_{44}\right\rangle\right)}+Q_{T \rightarrow T}\left(\frac{5}{2}+i k_{0 T} \sum_{j=1}^{N} A_{j} a_{j}\right)
\end{aligned}
$$

340 where $Q_{T \rightarrow T}$ is the transverse-to-transverse wave scattering factor for a triclinic polycrystal,

341 Ref. [13], and, due to the equalities (A1) (Appendix A), the LT factor is the same as in Eq.

342 (21). The stochastic asymptotes for the phase velocity and attenuation of a transverse incident 343 wave are:

344

$$
V_{T}^{S}=V_{0 T}\left[1+\frac{5\left(A_{T T}+B_{T T}+C_{T T}\right)}{16\left\langle c_{44}\right\rangle^{2}}+\frac{\left(A_{L T}+B_{L T}+C_{L T}\right)}{4\left\langle c_{44}\right\rangle\left(\left\langle c_{11}\right\rangle-\left\langle c_{44}\right\rangle\right)}\right]^{-1}
$$

$$
=V_{0 T}\left[1+\frac{5 Q_{T \rightarrow T}}{2}+\frac{\left(A_{L T}+B_{L T}+C_{L T}\right)}{4\left\langle c_{44}\right\rangle\left(\left\langle c_{11}\right\rangle-\left\langle c_{44}\right\rangle\right)}\right]^{-1}
$$

$$
\alpha_{T}^{S}=\frac{k_{0 T}^{2}\left(A_{T T}+B_{T T}+C_{T T}\right)}{8\left\langle c_{44}\right\rangle^{2}} \sum_{j=1}^{N} A_{j} a_{j}=k_{0 T}^{2} Q_{T \rightarrow T} \sum_{j=1}^{N} A_{j} a_{j}
$$

346 The analytical equations indicate that for the $L$ or $T$ incident wave the phase velocity at the

347 stochastic asymptote has no dependence on the TPC function and thus grain dimensions. The

348 stochastic attenuation asymptotes are proportional to the correlation length $\sum_{j=1}^{N} A_{j} a_{j}$, defined in Sec. II.A. One should note that for cubic polycrystals with equiaxed grains and an 
350 exponential TPC, asymptotes in Eqs. (14-25) degenerate to the forms as those given in Stanke and Kino [4].

\section{E. Far Field Approximation (FFA) model with generalized TPC for polycrystals with triclinic grains}

354 Further approximations, introduced in Ref. [13], can be made for the Green function of the

355 reference medium, eliminating the difficulty in computing Cauchy integrals. This is accomplished by adopting far field Green functions of scattered waves and the mean value theorem [13]. As for the Stanke and Kino model [4], the FFA model provides the dispersion equation in the spatial domain (quite the opposite from the SOA model where the dispersion equation, Eqs. (5-9), is given in the frequency domain). In this paper the FFA dispersion equations for a polycrystal with equiaxed grains are modified to include the generalized TPC, as in Ref. [23] but considering triclinic crystallites. The general dispersion equation of the FFA model for a longitudinal incident wave in a polycrystal with triclinic ellipsoidal grains and generalized TPC is:

364

$k^{2}(\mathbf{p})=k_{0 L}^{2}+k_{0 L}^{2}(\mathbf{p}) \sum_{N=L, T} \frac{k_{0 N}^{2} Q_{L \rightarrow N}}{\pi} \int \frac{\exp \left[i k_{0 N} r\right]}{r} w(\mathbf{r}) \exp (-i k \mathbf{p} \cdot \mathbf{r}) d^{3} \mathbf{r}$

365 where $\exp \left(i k_{0 N} r\right) / r$ is the far field Green function for a scattered wave $N$ [13], $Q_{L \rightarrow N}$ are scattering factors for triclinic crystallites (given in Appendix A and also introduced in previous sections); and $w(\mathbf{r})$ is the generalized TPC given by Eq. (4).

368 It is shown in [13] that for polycrystals with equiaxed grains and the exponential TPC function, the integral in the dispersion equation (26) may be analytically solved and for the generalized TPC the dispersion equation for a longitudinal incident wave becomes: 


$$
k^{2}=k_{0 L}^{2}+k_{0 L}^{2} \sum_{j=1}^{N} A_{j}\left(\frac{4 Q_{L \rightarrow L}}{\frac{k^{2}}{k_{0 L}^{2}}-\left(1+\frac{i}{k_{0 L} a_{j}}\right)^{2}}+\frac{4 Q_{L \rightarrow T}}{\frac{k^{2}}{k_{0 T}^{2}}-\left(1+\frac{i}{k_{0 T} a_{j}}\right)^{2}}\right)
$$

372 where the effective wavenumber $k$ has no direction dependence since the whole medium is statistically isotropic. Both attenuation and velocity are obtained solving this dispersion equation (27). The dispersion equation (27) is valid in all frequency ranges including the geometric region. Note the simplicity of the dispersion Eq. (27), compared to the much more computationally complex corresponding dispersion Eqs. (4-8) in the SOA model.

As discussed in reference [13], the dispersion equation modification mainly affects the near field part of the total scattering field on inhomogeneities. For this reason, the low frequency velocity limit of the FFA model is the velocity in the reference medium $V_{0 L}$, instead of $V_{L}$ given by Eq. (15). It was shown that a complete phase velocity $\left(V_{L}\right)$ dispersion curve is obtained by a constant shift using the SOA quasi-static velocity limit [13]. In displaying the velocity results in the following sections, we use the shift of the phase velocity by the constant obtained from Eq. (15):

384

$\left(V_{L}-V_{0 L}\right)=\Delta V_{L}=V_{0 L}\left[1+\frac{1}{2\left\langle c_{11}\right\rangle^{2}}\left(A_{L L}+B_{L L} / 3+C_{L L} / 5\right)+\frac{1}{2\left\langle c_{11}\right\rangle\left\langle c_{44}\right\rangle}\left(A_{L T}+B_{L T} / 3+C_{L T} / 5\right)\right]^{-1}-V_{0 L}$.

385 The dispersion equation of the FFA model with a generalized TPC function for a transverse 386 incident wave in polycrystals with equiaxed grains is:

$$
k^{2}=k_{0 T}^{2}+k_{0 T}^{2} \sum_{j=1}^{N} A_{j}\left(\frac{4 Q_{T \rightarrow L}}{\frac{k^{2}}{k_{0 L}^{2}}-\left(1+\frac{i}{k_{0 L} a_{j}}\right)^{2}}+\frac{4 Q_{T \rightarrow T}}{\frac{k^{2}}{k_{0 T}^{2}}-\left(1+\frac{i}{k_{0 T} a_{j}}\right)^{2}}\right),
$$


where $2 Q_{T \rightarrow L}=Q_{L \rightarrow T}$. The transverse phase velocity from the FFA model also requires a

389 constant shift [13], and the shift of the transverse phase velocity from the SOA model, Eq.

390 (18), is:

391

$\left(V_{T}-V_{0 T}\right)=\Delta V_{T}=V_{0 T}\left[1+\frac{\left(A_{T T}+B_{T T} / 3+C_{T T} / 5\right)}{4\left\langle c_{44}\right\rangle^{2}}+\frac{\left(A_{T L}+B_{T L} / 3+C_{T L} / 5\right)}{4\left\langle c_{11}\right\rangle\left\langle c_{44}\right\rangle}\right]^{-1}-V_{0 T}$

392 Since the FFA model uses further approximations in the SOA model, we compare it below

393 with the SOA, illustrating the FFA model's applicability.

394 F. Comparison of the FFA and SOA models for polycrystals with general 395 anisotropy grains

396 As indicated in Ref. [13], the FFA model has high computational efficiency and sufficient

397 accuracy; however, no comparisons were made in the past with the SOA model for crystallite

398 symmetries below cubic. Here, using the triclinic Copper Sulfate Pentahydrate (CSP)

399 polycrystal as an example, the FFA model is compared in Figure 1 with the SOA in all

400 frequency ranges for longitudinal and transverse waves. The Rayleigh and stochastic

401 asymptotes for the SOA model are also shown in the figure. The single crystal elastic

402 constants and density of CSP are given in Table I. The coefficients for the generalized TPC

403 function of the CSP polycrystal are discussed Sec. III.C.2.

404 In Figure 1 the top two figures are for the wave attenuation and the bottom two are for the 405 phase velocity (dashed curves are for the FFA model and solid lines are for the SOA). Two 406 branches of the dispersion equations are shown at high frequency for both models; the second 407 branch arises in the geometric region and may exist just in the near scattering field due to 408 extremely high attenuation. For the cubic polycrystals those branches were evaluated and 409 discussed in Refs. [13,19]. As follows from the comparison, the FFA model is generally in 410 excellent agreement with the SOA in the whole frequency range for both longitudinal and 
411 transverse waves. The results for the second branch, that appear in the geometric region,

412 show reasonable agreement between the FFA and the SOA models.

413 It is important to emphasize that the perturbed wave number $k$ in the dispersion equations $414(27,29)$ of the FFA model depends on the elastic covariances through two elastic scattering 415 parameters $Q_{M \rightarrow N}$ reducing the dependences on 21 elastic constants of triclinic crystallites 416 that appear in the SOA model to only two. The fact of the excellent agreement between the

417 two models indicates that the attenuation and velocity obtained from the SOA model are also 418 affected by this hidden combination of elastic constants. As was discussed in [13], this allows 419 us to obtain the equivalency of material systems for grain-scattering-induced attenuation and 420 velocity dispersion. PROPAGATION IN POLYCRYSTALS WITH TRICLINIC 423 GRAINS

424 This section addresses three-dimensional finite element (FE) simulations for longitudinal wave propagation in low symmetry polycrystals. We retain the triclinic polycrystalline CSP (Table I) example material, that was used in the preceding section, for these numerical studies, which provides complete generality for low symmetry materials. We compute the scattering induced attenuation and phase velocity, including the quasi-static phase velocity limit, for later comparison with the analytical models.

\section{A. Methodology of numerical modelling}

431 Recent developments in time domain FE simulation capabilities have enabled accurate 432 elastodynamic simulations of polycrystalline materials in realistic 3D cubic polycrystalline 433 sample volumes. This demonstrated capability has been reported by the authors in several 434 recent articles [21,23,25,34] and it provides a foundation for the present work focused on 
triclinic polycrystals. To avoid repetition, we provide just a brief summary of this method with focus on differences with and advances from the previous cases for cubic polycrystals.

437 The FE method uses grain-scale spatial representation to describe 3D polycrystalline materials. The spatial models, generated by Voronoi tessellation [27], are representative of realistic grain morphologies, and the grain geometry is equiaxed on average which is achieved by uniformly randomizing the Voronoi seeds in the three coordinate axes. In addition to the realistic representation of grain microstructures, the models are reproduced in significant sample volumes of large numbers of grains. This results in the capability of delivering a large number of random scattering events, which enables the description of scattering in a statistical sense as well as comparison with analytical models. Statistical description of wave scattering within polycrystals lies in the statistical description of the grain geometry, and for this aim, we use a two-point correlation (TPC) function as the statistical indicator of the spatial models. A detailed numerical procedure to measure TPC is presented in the following Sec. III.B.

Within the spatial model, each grain represents a crystallite and every grain is assigned the same material mass and stiffness properties. The material treated here is a triclinic material, namely copper sulfate pentahydrate (CSP [13]). It holds the lowest single-crystal symmetry and thus represents the case of general anisotropy. Although the grains adopt the same material, their crystallographic axes are uniformly randomly oriented and the model is thus statistically homogeneous and statically isotropic.

For the purpose of the 3D elastodynamic modelling, the models are discretized in both spatial and temporal domains [35]. For the spatial discretization, our previous study has shown that

457 either a structured or an unstructured mesh performs well under sufficiently fine 458 discretization [21]. This work adopts the former, i.e. structured mesh, which is advantageous 
due to the relatively good computational efficiency in performing the discretization. Such

460 discretization leads to the description of the spatial models by uniform 8-node linear "brick"

461 elements, the size of which is determined by the proper sampling of both wavelength and

462 grain structure. For these two length scales, a minimum of 10 samples is chosen to suppress

463 numerical errors as well as to well approximate grain structures. For temporal discretization,

464 a proper time step is defined by adopting the Courant-Friedrichs-Levy condition. This

465 condition designates the time step being at most equal to the smallest propagation time within

466 the discrete space, and it ensures incremental solution of the elastodynamic problem, stably

467 and accurately.

468 Loading and boundary conditions are configured to accommodate the desired wave 469 propagation mode, which is a longitudinal plane wave in this work. We place the FE model in 470 a Cartesian coordinate system as shown in Figure 2, with its outer surfaces being parallel to 471 the coordinate planes, and without loss of generality, we consider wave propagation in the $Z$ 472 direction. To simulate plane waves, symmetry boundary conditions (SBCs) are applied to the outer $X-Z$ and $Y-Z$ surfaces. On these outer surfaces, the SBCs constrain the displacement component in the direction perpendicular to the surface to be zero, and the FE model thus represents a repeatedly mirrored domain to simulate an infinite plate through which plane waves can be transmitted. A three-cycle toneburst signal is applied as a uniformly distributed force in the $Z$ direction to all of the nodes on the $X-Y$ surface at $Z=0$ and the transmitted signal is received by monitoring the displacements in the $Z$ direction on its opposite surface at $Z=L Z$, where $L_{Z}$ is the model dimension in the $Z$ direction. This configuration forms a pitch-catch 480 (through-transmission) setup and a typical example of coherent signals measured at these two surfaces is shown in Figure 2.

482 Upon completion of the above setups, the models can be solved using an elastodynamic time marching FE program [35]. To deliver modelling of wave scattering with better accuracy and 
less uncertainty, three spatial models are adapted to accommodate different frequency ranges.

The parameters of these three models are shown in Table II. At every frequency, it is necessary to use the average of multiple realizations (15 in this study) to obtain statisticallyconverged scattering results. These realizations, in principle, should be different in both grain structure and crystallographic orientation. This study, however, retains the same grain structures and achieves multiple realizations via re-randomizing crystallographic orientation of grains. It has been shown that this method preserves similar statistical variability as that which re-randomizes both grain structures and grain orientations [21]. Therefore, we are confident to use this method to save the intensive computational costs used to re-mesh the spatial models. The column labeled DOF records the total number of degrees of freedom in each model; the number of nodes is thus one third of this figure, and the number of elements is just a little less than the number of nodes.

\section{B. Quasi-static determination of velocity}

497 The quasi-static (or static) phase velocity limit (sometimes called pseudo-static), relates to the macroscopic effective elastic constants of homogenized polycrystals. The FE models as presented in Sec. III.A describe the polycrystals realistically, and thus the static velocities determined from them are helpful for the evaluation of methods for estimating effective elastic properties. Static velocities can be determined by static analysis to obtain the stiffness, but this requires the solution (equivalent to inversion) of the full stiffness matrix, which is beyond possibility with the large FE models. However, we have previously reported [23] that

504 the elastodynamic FE solver [35] can also be applied in a special way to determine a quasistatic velocity. This work further elaborates this methodology, and in particular reports on its accuracy.

507 Compared to the above outlined dynamic models, four changes are made to achieve quasi508 static modelling. The first one is the boundary condition, which assigns an additional 
constraint to the outer surface $Z=L_{Z}$ where the displacement in the $Z$ direction is fixed to

510 zero. The second one is the loading, which serves to simulate the state of static loading and 511 deformation while retaining the time marching elastodynamic solution methodology. This is

512 achieved by gradually applying a load to the FE model and keeping the final state of this load 513 as a constant. The load is applied as a uniformly distributed force in the $Z$ direction to all of 514 the nodes on the $X-Y$ surface at $Z=0$. Then, in order to allow the displacement field to settle 515 to a steady value after a sufficient period of time, material damping is defined for all of the elements. Although this steady state can always be reached eventually if any value of

517 damping is applied, the fastest way of achieving this is to apply critical damping to the first518 order mode of the FE model. The last change is to the measurement setup. Other than the 519 pitch-catch setup as outlined in dynamic modelling, the quasi-static modelling measures the excitation and transmitted signals on the same surface, i.e. $Z=0$, which forms a pulse-echo configuration. A typical example of time traces for loading and displacement is shown in Figure 3. Under such settings, it is easy to find that the constitutive equation can be simply expressed as $\sigma_{3}=C_{33} \varepsilon_{3}$, where $\sigma_{3}$ and $\varepsilon_{3}$ are the stress and strain in the $Z$ direction. $C_{33}$ is

524 the effective elastic constant in the $\mathrm{Z}$ direction of a homogenized polycrystal (due to isotropy 525 it equals to $C_{11}$ as discussed in Sec. IV.C). The stress and strain are given by $\sigma_{3}=F / A$ and $\varepsilon_{3}=u / L_{Z}$, where $F$ and $u$ are the applied force and displacement determined at steady state, $A$ is the area of the surface $Z=0$, and $L_{Z}$ the dimension of the model in the $Z$ 528 direction. Under such conditions, the longitudinal wave velocity in the $Z$ direction is $V_{L}^{S t}=\sqrt{C_{33} / \rho}=\sqrt{F l /(A u \rho)}$.

530 As an example, a homogeneous isotropic material (steel, with properties given in Table III) 531 has been used to check the accuracy of the FE quasi-static modelling to determine the static velocity. This model adopts the spatial model N11520 defined in Table II. The FEM static 
533 velocity obtained is $5944.0796 \mathrm{~m} / \mathrm{s}$; compared to the velocity of the selected material (Table

534 III), the relative error is only $0.0063 \%$. This indicates that the FE quasi-static methodology is

535 capable of achieving a highly accurate prediction of static velocities.

536 Similarly, the quasi-static velocity for the CSP polycrystal was determined for the spatial

537 model N115200 (Table II). The result is presented in Sec. VI for quantitative comparison

538 with analytical models.

539 C. Statistical representation of polycrystals: generalized two-point 540 correlation function

\section{1. 3D FEM two-point correlation function for simulated material systems}

542 The two-point correlation (TPC) function describes the probability of two points being in the

543 same grain (Sec. II). The numerical procedure for measuring this function from the 3D FE

544 material models has been discussed by the authors in Ref. [23]. The procedure involves a

545 random process of dropping two points, separated by a distance $r$, into the spatial model and

546 measuring the probability of the points being in the same grain. Repeated application of this

547 random process for incrementally varied $r$ produces a discrete TPC function $w(r)$. For

548 each $r$, the random process includes a significant number of random tests, one million in this

549 study, and thus gives a good statistical description of the spatial model with small 550 uncertainty. As previously [23], we exclude boundary grains by keeping the random points 551 away from the model boundaries. These FEM TPC functions for the three spatial models 552 (N115200, N11520 and N16000) are shown by points in Figure 4.

\section{Approximation of 3D FEM TPC function by generalized TPC series}

554 The numerically-obtained spatial two-point correlation function (TPC) for the FEM material systems deviates considerably from the exponential correlation function $\exp (-r / a)$. In order to incorporate the measured FE TPCs into analytical models, we fit these discrete TPC functions by exponential series (Sec. II.A). We call this analytical representation the 
generalized two-point correlation function, as it is different from a classical description of polycrystals using single-term exponential functions [4,5]. Compared to the classical treatment, it has been shown [23] that the incorporation of the generalized TPC into analytical models gives better agreement with the FE modelling; it thus enables better evaluation of the suitability of the approximations included in the analytical models. For

563 clarity and relevance to this present work, we show only the generalized TPC in Figure 4; readers interested in the comparison with the single-term exponential are referred to our previous work in [23].

Since the TPC functions for the three spatial models are almost identical (Figure 4), we take the average of these three TPCFs and treat it as the TPC function from the FEM material model. The coefficients of the generalized analytical TPC function are determined by the non-linear least square fitting of the averaged TPC data. The fitting was performed with constraint Eq. (2) and slightly relaxed constraint (3), using eight terms $(n=8)$ in the exponential series. The TPC coefficients are given in Table IV. While the non-linear least square fitting is stable in general, the fitting becomes unstable for a larger number of terms.

The value of the coefficients $A_{i}, a_{i}$ depend on number of terms $N$ in the series and conditions coefficient and phase velocity depend on the integral characteristics of the generalized TPC and total accuracy of the fitting of the actual material TPC. We discuss this issue further in Sec. IV.D. The fitted generalized TPC function, $w(r)$, is shown by a solid line in Figure 4; very good agreement is observed between the fitted TPC and the data except for largest $r$, where the $w(r)$ function is very small.

580 As discussed in Sec. II.A, the line intercept is $a_{l}=-\left[w^{\prime}(r=0)\right]^{-1}$. The measured TPC slope at the origin of the 3D FEM TPC is -2.893 , which corresponds to a mean line intercept 
$a_{l}=0.346 \mathrm{~mm}$. The mean grain diameter [36] determined from the 3D grain size distribution of

583 the CSP polycrystal (shown in Table II) is $0.5 \mathrm{~mm}$, which corresponds to a mean grain radius

$584 a_{G}=0.25 \mathrm{~mm}$, indicating a difference with the mean line intercept.

\section{IV. COMPARISON OF THE ANALYTICAL AND FE MODELS}

586 We now proceed to compare the predictions of the analytical models (the FFA and SOA)

with those of the FEM simulations on attenuation and phase velocity. The quasi-static velocity from the SOA model is also compared with the FEM results obtained in this work and the other static velocity bounds developed in the literature. Finally, we compare the attenuation and velocity results obtained by the analytical and the FE methods for the triclinic polycrystal that has been studied in this work, and also with the results from our previous studies of cubic polycrystals [21,23]. This allows us to illustrate the effect of the elastic scattering factors on the discrepancy between the analytical and the FE model results (for cubic polycrystals these scattering factors are proportional to the crystallite anisotropy).

\section{A. Attenuation coefficients for CSP polycrystal}

\section{Attenuation coefficient: the SOA, FAA and FE models}

597 The normalized longitudinal attenuation coefficient obtained by the 3D FEM and the analytical, SOA and FFA models, are shown in Figure 5(a) versus nondimensional frequency $2 k_{L} a_{l}$. The results are for the polycrystalline CSP FEM material system with triclinic equiaxed grains; the coefficients for the generalized TPC function are given in Table IV. The calculations via the SOA and FFA models are shown by lines; the FEM results (points) are represented by different symbols for the three material models (Table II). The Rayleigh limit and stochastic asymptotes from Eqs. (15) and (21) are also depicted. The analytical curves are not extended to the geometric region, since the FEM data are not available for comparison in this regime due to extremely high attenuation and the level of discretization required. 
606 Generally, the quality of agreement between the analytical and 3D FEM results is

607 remarkable. In particular, the analytical models (both the SOA and FFA) have excellent

608 agreement with the 3D FEM in the Rayleigh region and the transition region to the stochastic

609 regime; the FFA model is almost indistinguishable from the SOA in the whole frequency

610 range. The analytical and the FEM results approach the Rayleigh asymptote at very low

611 frequency and the stochastic asymptote at high frequency. One significant observation is that

612 in the transition frequency range the "hump" behavior displayed by the analytical models

613 agree well with the FEM results. This hump is a unique feature for the longitudinal wave

614 attenuation [4] and is physically explained by the dominant scattering mode transition: from

615 the longitudinal-to-transverse wave scattering in the Rayleigh regime to longitudinal-to-

616 longitudinal wave scattering in the stochastic region. The results show smooth transition from

617 the fourth power of frequency in the Rayleigh region to second power dependence in the

618 stochastic region. The frequency behavior in the transition range significantly deviates from

619 that for the Rayleigh or stochastic asymptotes. Note that in the unique case, reported

620 elsewhere [6,22,31], when the polycrystal is formed by spherically- shaped grains having

621 nearly the same radius, oscillations for attenuation in the transition regime may take place.

622 In particular Ryzy et al. [22] found strong dependence of the attenuation in this regime on the

623 TPC function (especially scattering into the transverse wave, $\alpha_{L T}$ attenuation component).

624 The nonmonotonic behavior of the longitudinal-to-transverse attenuation component with

625 frequency and a strong dependence on the TPC in the transition region are demonstrated

626 below in Sec. D.

627 To quantify the discrepancy of attenuation results between the analytical and FE models for

628 the CSP polycrystal shown in Figure 5(a), the root-mean-square deviation (RMSD) has been

629 calculated for the whole frequency range and is shown in Table V (last row); it is normalized

630 by the FEM attenuation. The RMSD of the attenuation coefficients between the SOA model 
and 3D FEM is $9.44 \%$, and that for the FFA model is $7.77 \%$. It is interesting that the discrepancy between the SOA and FEM for the triclinic CSP is smaller than those for Inconel and copper and $\mathrm{A}=2.4$ materials, this is discussed in Sec. IV.D.

\section{The Born approximation and application to the backscattering coefficient}

635 In this section, we briefly discuss the extension of the conclusions on the agreement between

636 the analytical and the FE models to the backscattering coefficient. Since we have already

637 developed and discussed the analytical attenuation models, the simplest way to obtain the

638 backscattering coefficient is from the wave attenuation coefficients in the Born 639 approximation $[12,20]$.

640 Figure 5(b) compares the FEM, SOA and Born approximation results for the CSP polycrystal; the latter is shown by a dashed line, which replaces that for the FFA model in part (a) of the figure. The Born approximation has in general very good agreement with the

643 SOA; however, at higher frequencies (above $2 k_{L} a_{l}=20$ in Figure 5.b), where forward

644 multiple scattering becomes stronger, it departs from the SOA model and follows the 645 stochastic asymptote. This departure is associated with the start of the transition to the 646 geometric region; Figure 1 clearly shows the separation of the SOA and the FFA models 647 from the stochastic asymptote and the transition from the stochastic to the geometric region.

648 In this frequency range the SOA attenuation coefficients due to partial accounting of the 649 forward multiple scattering increases more. For cubic materials this was noted in [4] and the 650 deviation of the Born approximation from the FFA model at high frequencies was discussed 651 in [13]. The RMSD between the Born approximation and the FEM is $11.47 \%$.

652 Instead of using a single integral representation (the second term in Eq. (5), taking $k=k_{0 L}$ )

653 for the Born approximation, we consider the attenuation coefficients in the spherical 654 coordinate system that represents an integration over the wave scattering in all angle 
directions. Therefore, the angle-dependent mode scattering coefficients may be obtained directly from the integrand of the attenuation coefficients $[12,20]$. Such obtained coefficients are equal to those known in the literature.

658

From the Born approximation for the wavenumber, Eq. (13), substituting the imaginary part of the mass operator $m_{L \rightarrow L}\left(k_{0 L}\right)$, we have for the $\alpha_{L \rightarrow L}$ component of the total attenuation coefficient $\alpha_{L}$ in the spherical coordinate system:

661

$$
\alpha_{L \rightarrow L}=-\frac{\operatorname{Im} m_{L \rightarrow L}\left(k_{0 L}\right)}{2 k_{0 L} V_{0 L}^{2}}=\frac{k_{0 L}^{4}}{4 \pi \rho^{2} V_{0 L}^{4}} \int_{0}^{2 \pi} \int_{0}^{\pi} \sum_{j=1}^{N} A_{j} a_{j}^{3} f_{L L}\left(k_{0 L}, a_{j}, x, 1\right) \sin \theta d \theta d \phi .
$$

662 Here $x=\cos \theta$, and one needs to consider the double integrals over two angles $\phi$ and $\theta$ even

663 for the equiaxed grains to account in the integrand for all scattering angles. Taking the 664 integrand in Eq. (31) and considering $\theta=\pi(x=-1)$ and taking the attenuation coefficient 665 for the energy flow [12,20] (thus factoring the integrand by 2), we have for the 666 backscattering coefficient:

$667 \quad \eta_{L \rightarrow L}=\frac{Q_{L \rightarrow L} k_{0 L}^{4}}{4 \pi^{2}} \sum_{j=1}^{N} A_{j} \frac{8 \pi a_{j}^{3}}{\left[1+4 k_{0 L}^{2} a_{j}^{2}\right]^{2}}$

668 When Eq. (32) is reduced to a single exponential TPC the backscattering coefficient (32) is 669 equivalent to that in Ref. [12] for triclinic grains, and for hexagonal and cubic symmetries to 670 that of $[20,46]$, where a notation for factor $Q$ relates to the elastic scattering factor $Q_{L \rightarrow L}$ of

671 this work as $Q_{L \rightarrow L}=4 \pi^{2} Q$.

672 The model approximations in Eq. (32) for the backscattering coefficient are the same as for 673 the SOA Born approximation Eq. (31). Therefore, the conclusions on agreement with the 3D 674 FE computations are similar to those for the attenuation coefficients, Figure 5(b). Indeed 
recent comparison of the cubic polycrystals of the modeled backscattering amplitude and the 3D FEM [26] indicate reasonable agreement.

\section{B. Phase velocity for CSP polycrystal}

678 Phase velocity data from the analytical models, SOA and FFA, are compared with the FEM 679 in the normalized frequency domain in Figure 6, where the phase velocity from the FFA 680 model is corrected using Eq. (21). The Rayleigh and stochastic limits for phase velocity, Eqs. 681 (15) and (21), are plotted by dotted lines, they are independent of frequency. One can see that 682 the SOA model approaches the Rayleigh limit at very low frequency and the stochastic asymptote at high frequency (the transition to and the geometric region are not shown). The Voigt velocity $V_{0 L}$ in the reference medium is slightly above the stochastic asymptote.

The velocities obtained by the analytical SOA and FFA models are in excellent agreement with the FEM results from the Rayleigh to the stochastic regimes. The largest discrepancy takes place near the normalized frequency 6.34 with an absolute deviation $27 \mathrm{~m} / \mathrm{s}$, which is $0.34 \%$ in the relative difference with the FEM velocity as the reference. This occurs at the end of the transition region that is very sensitive to the accuracy of the TPC (refer to Sec. IV.A.1 for extensive discussion of the transition region). The RMSD between the FE and SOA models for the whole frequency range is $0.014 \%$, and the RMSD between the FEM and the corrected FFA for the whole frequency range is $0.021 \%$. Comparing to the attenuation RMSD (Table V), this discrepancy is negligible. We can see the agreement between the SOA and the corrected FFA is also excellent, except at highest frequency (in the vicinity of $\left.2 k_{L} a_{l}=10\right)$. The part of the discrepancy between the analytical models and the FEM may be attributed to the SBC effect in the FE model, our investigation indicates that the SBC effect on the velocity may result in a RMSD of $0.05 \%$, which means that the discrepancy between analytical models and FEM falls into the error range caused by the SBC effect (as briefly discussed in Sec. IV.D). 
700 The velocity dispersion manifests itself in the transition range between the Rayleigh and

701 stochastic regimes. It changes about $2 \%$ in the nondimensional frequency $2 k_{L} a_{l}$ ranging from

702 about 3 to 10 . Most of this change occurs between the Rayleigh and the stochastic velocity

703 limits, which can easily be estimated from the analytical equations $(15,21)$. To the

704 knowledge of the authors, this account for the ultrasonic velocity dispersion in polycrystals is

705 mostly absent in experimental ultrasonic studies when compared with the static or low

706 frequency resonance measurements of the elastic constants. The fact that the measured

707 ultrasonic velocity should approximately vary between the Rayleigh and the stochastic

708 theoretical limits may be very useful in experimental ultrasonic studies, since the

709 approximate bounds of the phase velocity change allow simple estimates for arbitrary

710 crystallographic symmetries of the grains, Eqs. $(15,21)$.

\section{C. Quasi-static limit of phase velocity for CSP polycrystal}

712 As another important topic, we address here a discussion of the quasi-static velocity limit

713 obtained from the SOA model, which provides effective elastic constants of homogenized

714 random polycrystalline aggregates. We treat the velocity from the SOA model at a frequency

715 constant 0.01 (Figure 6) as its static velocity representation since it asymptotically

716 approaches its low frequency analytical asymptote, which is constant. For comparison with

717 the SOA model, the static velocity from the FEM is also shown in Figure 6 (the solid square

718 point). Those two static velocities are reasonably close (see the velocities given in Table VI),

719 and the relative difference between the SOA and the FEM is $0.08 \%$ with the FEM result as a

720 reference. The analytical quasi-static limits, Eqs. $(15,18)$, derived from the SOA model, are

721 new; they are suitable for all symmetry classes. They were known only for cubic crystallite

722 symmetry as given in Stanke and Kino [4] (the misprints in equations [4] for those limits

723 were corrected in [13]). 
724 We have performed a systematic comparison of the static velocities obtained in this study and those calculated from information available in the literature for polycrystals with triclinic constituents that resulted from the recent homogenization models [37,38]. Well known bounds, the Voigt-Reuss-Hill bounds [39] are also available for comparison. The HashinShtrikman (H-S) upper and lower bounds and the self-consistent (SC) velocity are calculated for the CSP polycrystal in terms of the open source program in Refs.[37,38] and plotted in Figure 6 (see H-S U, H-S L and SC static). The approaches reported in Refs. [37,38] are essentially numerical methods. Optimal bounds for bulk and shear moduli were determined numerically by the variational method in Ref. [40], while the approach with the transcendental equation of SC, given in Ref. [37] for bulk and shear moduli, was solved by an iterative method. One can observe from Figure 6 that our quasi-static velocities (obtained by the SOA and the FE models) fall between the H-S upper and lower bounds and they are very comparable to the SC velocity.

To further facilitate quantitative comparison, Table VI shows all static velocity bounds $V_{L}^{S t}=\sqrt{C_{11} / \rho}$ calculated by us, namely the Voigt-Reuss-Hill velocities, the H-S bounds and the SC velocity, as well as those obtained in this work (the SOA, FEM, FFA). The calculated

740 effective elastic constant $C_{11}$ of the macroscopically isotropic homogenized polycrystal is

741 shown in the first row of the table, the density $\rho$ is from Table I. The calculated shear elastic constant, $C_{44}$, is given in the third row for completeness. The elastic constants $C_{11}$ and $743 C_{44}$ obtained from the quasi-static velocity limits, SOA and Eqs. $(15,18)$, are within the H-S 744 bounds and close to the self-consistent values.

745 As expected, the H-S bounds provide a narrower velocity variation range than the Voigt746 Reuss bounds. Interestingly, the SC velocity is slightly higher than the Hill average (an 747 average of the Voigt and Reuss bounds), and the static velocity from the SOA model is 
748 almost identical to the SC velocity. Quasi-static velocities from the 3D FEM and the 749 analytical equation of the SOA at the Rayleigh limit, Eq. (15), are also in good agreement 750 with the SC velocity. Remarkably, as follows from Table VI, the SC static velocity, the SOA

751 model limit at $2 k_{L} a_{l}=0.01$, the quasi-static velocity from Eq. (15) (or Rayleigh limit velocity), and the quasi-static FEM velocity rounded to three digits all equal 4.79 and are within the HS bounds. Therefore the analytical static velocity Eq. (15) obtained in this study provides an accurate simple method to calculate the quasi-static velocity for aggregate crystallites of different crystallographic symmetries.

756 Note that in the recent numerical study [41] of static homogenization of polycrystalline material, it was shown that the number of crystallites in the numerical model can affect the effective elastic constants of the homogenized medium. It was shown [41] that this influence depends on the universal anisotropy factor. In the extreme case in Ref. [41] (the universal anisotropy factor up to 1.82 ) 5,000 grains were necessary to reach statistically steady effective moduli. For all material systems in this work and our previous studies $[21,23]$ the universal anisotropy factors are smaller than 1.82 (see Table VI), and the grain numbers in the FE material models are far larger than 5,000 (the minimum number is 16,000 in our studies). Thus, for the FEM quasi-static velocities in this work and previous work [23] the grain number effect is negligible.

\section{Comparison of results for triclinic CSP and cubic polycrystals}

Finally, we compare the attenuation coefficients for six previously studied cubic material systems [21,23] and the CSP triclinic material system. The results are summarized in Figure 7 for the SOA model (a) and the FE model (b). In this case the attenuation coefficients and the wavenumber are normalized by the line intercept $a_{l}$ instead of the $a_{G}$, as in [21,23], where $a_{G}$ was obtained from the volumetric grain size distribution. This is done because the line 
intercept $a_{l}$ is usually the grain characteristic that may be measured in experiments $[30,32]$.

773 For this reason, the attenuation curves for all cubic systems were replotted to make them consistent with that for the CSP. Adopting different mean grain size only shifts the curves

775

776

777

778

779

780

781

782

783

784

785

786

787 (both for the FEM and analytical model) in the normalized frequency domain but does not affect the relative difference between the FEM and the analytical models; the agreement between 3D FEM and the analytical model presented in Ref. [23] does not change.

The material systems compared here have different universal anisotropic indices of grains that are given in Table VII. The universal anisotropy factor $A^{U}$, equivalent anisotropy factor $A^{E}$, and anisotropy index $A^{L}$ for each material are calculated using [42], [43]; note that for cubic symmetry the equivalent anisotropy factor $A^{E}$ equals the Zener anisotropy factor $A=2 c_{44} /\left(c_{11}-c_{12}\right)$, which is given in the first column of Table VII. All three anisotropy factors in Table VII are consistent with each other; copper has the largest anisotropy, while the anisotropy factors of the triclinic CSP crystal are a bit smaller than those for the $A=2.4$ material. We discuss further below that scattering in triclinic polycrystals is not controlled by the anisotropy index.

We gauge the deviations between the analytical and the 3D FEM attenuation coefficients by the RMS discrepancies in the frequency range of the data availability. In addition to the RMSD for the longitudinal attenuation coefficient for the CSP polycrystal, discussed in Sec. VI.A (the last row in Table V), the RMSD for six cubic polycrystals evaluated in Refs.[21], [23] also are given (the RMSD is normalized by the FEM attenuation). The universal anisotropy factor $A^{U}$, scattering factors $Q_{L \rightarrow M}$, and attenuations, predicted by the SOA model at low $2 k_{L} a_{l}=1$ and high $2 k_{L} a_{l}=20$ frequencies, are also shown in Table $\mathrm{V}$ for all materials studied. 
795 From Table V, one may conclude that generally, for cubic materials (first six rows), the 796 attenuation coefficients for the SOA and FFA models increase with the anisotropy factor $A^{U}$.

797 This is not the case for the triclinic CSP polycrystal, which has the highest attenuation but has 798 anisotropic index between the $\mathrm{A}=1.4$ and $\mathrm{A}=1.8$ materials. For all material systems the 799 attenuation coefficients increase with the scattering factors $Q_{L \rightarrow L}$ and $Q_{L \rightarrow T}$ resulting in 800 higher and higher attenuation in the Rayleigh and stochastic regions (see the last two columns 801 in Table V and Figure 7(a,b) for the SOA and FE models). These two scattering factors $Q_{L \rightarrow L}$ 802 and $Q_{L \rightarrow T}$ are explicitly related to the quadratic equations of elastic constants (Appendix A 803 and Ref. [13]). The longitudinal wave scattering factors $Q_{L \rightarrow L}$ and $Q_{L \rightarrow T}$, for the material 804 systems with crystallographic symmetry lower than cubic, cannot be systematically related to 805 any of the three universal anisotropy indices given in Table V. It is the opposite for the 806 transverse wave attenuation coefficient [44], where the scattering factor $Q_{T \rightarrow T}$ was 807 approximately linked for any crystallographic symmetry to the equivalent anisotropy index $808 A^{E}$, similar to that for cubic crystallographic symmetry.

809 In quite the opposite manner, regarding the attenuation coefficient, the RMSD values 810 between the analytical and the FE models in general increase with the anisotropy index $A^{U}$ 811 for all material systems including the triclinic CSP. Therefore, the RMSD behavior seems not 812 to be controlled by the level of material perturbations (related to the scattering factor $Q_{L \rightarrow L}$ 813 [4,20]). This indicates the possibility that a higher level of discretization is needed in the FE 814 model for high anisotropy grains to account for the stronger change of elastic properties with 815 angle (ideally a discretisation convergence study could be used to check this, but this is 816 currently not practical to achieve within the available computational resources; it may be 817 attempted in a future study as computing resources continue to grow). One can also conclude 818 from Table $\mathrm{V}$ that the RMSDs for the SOA and FFA models are very close for all $38 / 76$ 
819 polycrystals. Overall, the agreement between the analytical models (SOA and FFA) and the 820 FEM is very good.

821 The attenuation coefficient for the triclinic CSP is slightly higher in the Rayleigh and stochastic regions than those for cubic Inconel and copper (Table V), indicating again the

823 important effect of the elastic scattering factors $Q_{L \rightarrow M}$. However, in the transition region (the 824 hump region) the behavior is more complicated, as can be seen in Figure 7. For a more 825 detailed discussion below, we have replotted the attenuation coefficient for those three 826 materials on a larger scale, in Figure 8(a). Figure 8(b) provides the TPC functions for those 827 materials; the TPCs for Inconel and copper are indistinguishable.

828 The stochastic regime: The CSP has the strongest L-L scattering factor $Q_{L \rightarrow L}$, which 829 dominates the scattering in the stochastic region. Specifically, the attenuation coefficient in 830 the stochastic region relates to $Q_{L \rightarrow L}$ as:

831 $\alpha_{L}^{S}=k_{0 L}^{2} Q_{L \rightarrow L} \sum_{j=1}^{N} A_{j} a_{j}=k_{0 L}^{2} Q_{L \rightarrow L} a_{C L}$

832 From this equation, due to having the largest $Q_{L \rightarrow L}$ the CSP has the largest attenuation in the 833 stochastic regime, given approximately the same TPC functions for CSP, Inconel and copper, 834 Figure $8(\mathrm{~b})$. Actually the $a_{C L}$ for the CSP is slightly smaller, as shown in Table VIII, but $835 Q_{L \rightarrow L}$ compensates for this difference. For the attenuation in the stochastic region, Figure $8368(\mathrm{a})$, we have $\alpha_{C S P}>\alpha_{\text {Inconel }}>\alpha_{\text {Copper }}$, which is consistent with the related sequence of the $837 Q_{L \rightarrow L}$ factors (but is not consistent with the anisotropy factors).

838 The Rayleigh regime: The L-T elastic scattering factors $Q_{L \rightarrow T}$ govern the scattering strength 839 in the Rayleigh region for the attenuation component $\alpha_{L \rightarrow T}^{R}$. For the CSP, Inconel and copper 
materials the $Q_{L \rightarrow T}$ factors are comparable; however, the attenuation coefficients in the

841 Rayleigh region (for both the SOA and the FEM) obey the inequality: $\alpha_{C S P}>\alpha_{\text {Copper }}>\alpha_{\text {Inconel }}$,

842 Figure 8 and Table $\mathrm{V}$ at $2 k_{L} a_{l}=1$. This is a result of the combined effect of the factors $Q_{L \rightarrow L}$,

$843 \frac{V_{0 L}^{3}}{V_{0 T}^{3}}$ and $V_{g}^{R}=\sum_{j=1}^{N} A_{j} a_{j}^{3}$ appearing in the Rayleigh asymptote:

$844 \quad \alpha_{L \rightarrow T}^{R}=4 Q_{L \rightarrow T} k_{0 L}^{4} \frac{V_{0 L}^{3}}{V_{0 T}^{3}} \sum_{j=1}^{N} A_{j} a_{j}^{3}=4 Q_{L \rightarrow T} k_{0 L}^{4} \frac{V_{0 L}^{3}}{V_{0 T}^{3}} V_{g}^{R}$,

845 which implies that the $\alpha_{L \rightarrow T}^{R}$ is also related to the Poisson's ratio. To fully explain our

846 observations, the Poisson's ratio, $\frac{V_{0 L}^{3}}{V_{0 T}^{3}}, V_{g}^{R}=\sum_{j=1}^{N} A_{j} a_{j}^{3}$ and $\sum_{j=1}^{N} A_{j} a_{j}$ for the CSP, copper and

847 Inconel are given in Table VIII. As noted before, the TPC for these materials are not

848 identical, Figure 8(b), so we have to account for the TPC effect on the grain volume terms

$849 V_{g}^{R}=V_{g}^{e f f}=\sum_{j=1}^{N} A_{j} a_{j}^{3}$, Table VIII. The products of $\frac{V_{0 L}^{3}}{V_{0 T}^{3}}$ and $\sum_{j=1}^{N} A_{j} a_{j}^{3}$ are comparable for the CSP and

850 copper indicating that those attenuation coefficients are larger than that for Inconel in the

851 Rayleigh regime, which is consistent with Figures 7,8 including the FEM results.

852 The transition region: One unique observation for Inconel is that its transition region for the attenuation coefficient starts at relatively high frequency $2 k_{L} a_{l}$ and the attenuation coefficient

854 is slightly higher than those for copper and the CSP; it is especially pronounced in the FEM results. This is elucidated by examining in Figure 9 the attenuation components $\alpha_{L T}, \alpha_{L L}$,

856 which have been calculated using the SOA's Born approximation with the generalized TPCs

857 shown in Figure 8(b). The longitudinal-to-transverse wave scattering $\alpha_{L T}$ for Inconel dominates the attenuation coefficient (Figure 9) significantly further into the transition region than that for the CSP, resulting in the higher total attenuation coefficient. Only with 

frequency increase does the total attenuation coefficient for the CSP become slightly larger and it overtakes that for the Inconel with frequency increase further into the stochastic region.

862 This frequency delay of the transition region is essentially affected by the combination of the 863 factors in Eq. (34). Figure 9 also amplifies the point made in Sec. IV.A.1 on the strong nonmonotonic dependence of the $\alpha_{L T}$ attenuation component on frequency and its strong

865 dependence on the TPC function in the transition regime.

866 As we have discussed above, the theoretical solutions for the attenuation and velocity depend 867 on the integral characteristics of the generalized TPC. The correlation length is defined in 868 Sec. II.A as $a_{C L}=\int_{0}^{\infty} w(r) d r=\sum_{j=1}^{N} A_{j} a_{j}$ and this TPC characteristic plays a major role in 869 the stochastic region. The volume TPC characteristic $V_{g}^{\text {eff }}=\int_{V} w(r) d V=8 \pi \sum_{j=1}^{N} A_{j} a_{j}^{3}$ dominates scattering in the Rayleigh regime. Thus, it is interesting to compare those characteristics for the generalized TPC and those numerically obtained by integrating the actual material TPC. Numerical values for the CSP FEM material system are given in the two last rows of Table

873 VII. The numerical $a_{C L}$ and $V_{g}^{\text {eff }}$ are in reasonable agreement with those for the generalized 874 TPC; the deviations between these values indicate that the expected contribution to the analytical attenuation coefficient from inaccuracies of the actual TPC fitting may contribute about $2 \%$ in the Rayleigh regime and about $5 \%$ in the stochastic regime. In addition to the approximations used in the SOA analytical model that affect the RMS discrepancy between the SOA and the FE models, other error sources may contribute to the discrepancy. One source of the discrepancy is the effect of symmetry boundary conditions

880 (SBC) employed in the 3D FEM (this question will be addressed in detail elsewhere). 881 Physically, the SBC doubles the grain size for grains that lie on the model boundaries in the 3D FE material model and therefore slightly increases scattering in the FE model. This effect 
is not accounted for in the analytical models by the generalized TPC; this is partially because

884 in the analytical model it is assumed that the larger boundary grains are distributed spatially

885 homogenously, instead of at the SBC as in the FEM. Calculations show that the SBC effect 886 contributes about 5\% into the RMSD for the whole frequency range. Additionally, the second 887 source of error is the accuracy of the generalized TPC fitting of the actual material TPC that 888 could produce an error of about $1 \%$.

\section{SUMMARY AND CONCLUSIONS}

890 The second order approximation (SOA) and the 3D finite element (FE) models are developed

891 for elastic wave propagation in polycrystals with general crystallographic symmetry

892 (triclinic) of equiaxed grains, aiming for determination of ultrasonic attenuation and phase velocity. For the 3D FEM material systems studied their two-point spatial statistics are not well represented by the exponential TPC. For this reason, the analytical models employ the generalized two-point correlation function (TPC) that is obtained by exponential series fitting of numerical TPCs for the FE material systems. As of the type of Stanke and Kino's ([4]) model, developed for cubic polycrystals, the SOA model is applicable in the whole frequency range and accounts for forward multiscattering effects. The analytical Rayleigh and stochastic asymptotes for attenuation and phase velocity have been derived and compared with the FE

900 simulations. Also, systematic comparison of the SOA, FEM and the far field approximation 901 (FFA) model, has been performed for triclinic polycrystals (the FFA [13] has been extended 902 to include the generalized TPC function).

903 The results of comparison of the FE and analytical models for triclinic polycrystals indicate 904 the validity of the analytical model assumptions, such as replacement of the polycrystal by a 905 continuous elastic medium with random fluctuations of the elastic tensor and the assumption

906 of weak scattering in the further analytical model approximations. This was demonstrated for 

the triclinic CSP with a relatively large perturbation factor $\varepsilon^{2}$, which is defined in references

$908[4,20]$ and is equivalent to the elastic scattering factor $Q_{L \rightarrow L}$ (for the $Q_{L \rightarrow L}$ values see Table V). 909 This is of great significance because it provides a theoretical foundation for further 910 development of ultrasonic characterization methods of polycrystals with low symmetry grains

911 like $\alpha / \beta$ colonies in $\mathrm{Ti}$ alloys. To achieve a more general perspective, systematic 912 comparison of the current results for the triclinic CSP and of our prior results [21,23] for 913 different cubic polycrystals was performed covering a large range of perturbation factors and 914 anisotropy indices (Table V).

915 The following key conclusions were reached:

916 1) In general, for the triclinic CSP polycrystal, good agreement for ultrasonic attenuation and 917 phase velocity is obtained between the SOA and the FE models when the fitted generalized 918 TPC function for the FE material system is used in the SOA model; the normalized RMS 919 difference for attenuation is below $10 \%$ and for phase velocity is $0.014 \%$.

920 2) For the triclinic polycrystal, the longitudinal wave attenuation obtained by the far field 921 approximation (FFA) model shows good agreement with that of the SOA and that of the FE 922 models. The phase velocity obtained from the FFA agrees well with both the SOA and the 923 FEM results in all frequency ranges investigated after a constant shift by the quasi-static 924 velocity limit determined from the SOA model.

925 3) It is confirmed by the FEM computations and by comparison of the FFA and SOA models 926 that the longitudinal wave attenuation in statistically isotropic polycrystals formed by triclinic 927 crystallites depends on the 21 crystallite elastic constants mostly through their combination 928 expressed by the two scattering elastic factors $Q_{L \rightarrow L}$ and $Q_{L \rightarrow T}$, obtained from the FFA model 
between the SOA the FFA models, shown in this work and in Refs. [13,23,44], indicates the universality of this relation.

932 4) The attenuation coefficient obtained from the analytical and the FE models generally increases with increase of the elastic scattering factors $Q_{L \rightarrow L}, Q_{L \rightarrow T}$ for all of the polycrystals

934 that were studied. For cubic polycrystals they also increase with the crystallite anisotropy

935 factor $A$; this is because the elastic scattering factors $Q_{L \rightarrow L}, Q_{L \rightarrow T}$ depend on the anisotropic

936 coefficient $c$ squared [13]. However, the triclinic CSP polycrystal does not follow this

937 behavior on the anisotropy index and both scattering factors $Q_{L \rightarrow L}, Q_{L \rightarrow T}$ should be considered

938 to judge the level of stochastic material perturbations. In particular, we found that, while the

939 universal anisotropy indices for cubic $\mathrm{Cu}$ and $\mathrm{Ni}$ crystallites are significantly larger than

940 those for triclinic CSP crystallites, the scattering-induced longitudinal attenuation for the CSP

941 polycrystal is greater than or nearly equal to, depending on the frequency range, the

942 attenuation in cubic $\mathrm{Cu}$ or $\mathrm{Ni}$ polycrystals, Table $\mathrm{V}$. This is different from the transverse

943 wave attenuation depending mostly on the $Q_{T \rightarrow T}$ elastic scattering factor, which increases with

944 the universal anisotropy indices independently of the crystallite symmetry [44].

945 5) For all polycrystals studied the normalized discrepancy between analytical models and the

946 FEM (RMSD) generally increases with the universal crystallite anisotropy index $A^{U}$ for

947 longitudinal attenuation (with the RMSD increase from $7 \%$ for $\mathrm{Al}$ to about $14 \%$ for $\mathrm{Cu}$ and

948 Inconel; for the CSP the RMSD is about 9.4\%, Table V).

949 6) The ultrasonic phase velocity is nearly constant with normalized frequency in the Rayleigh 950 and stochastic regions. For those regions new analytical SOA velocity limits are obtained for 951 triclinic polycrystals; the limits are independent of the TPC. The phase velocity varies 952 between those limits while transiting between the Rayleigh and stochastic frequency regions.

953 Those limits provide simple estimates of the possible range of the experimental velocity 
954 dispersion (see the example for $\mathrm{Cu}$ in [23]) and may simplify understanding of the 955 experimental results when comparing different acoustic and static measurements of the 956 elastic constants.

957 7) The SOA quasi-static velocity limit for CSP is between the Hashin-Shtrikman bounds for 958 triclinic polycrystals and agrees to within nearly four digits with that obtained by the FEM 959 and the self-consistent static velocity estimate (Table VI).

\section{ACKNOWLEDGMENTS}

961 The authors appreciate the contribution of Dr. A. Van Pamel who performed preliminary

962 FEM computations for polycrystals with triclinic crystallites and for many different insights

963 on numerical modelling, and to Dr P Huthwaite who provided valuable assistance with the

964 development and successful execution of the very large FE simulations. The authors are also

965 thankful to Dr Yilong Zhang from OSU for useful discussions of asymptotes. G. Sha and S.I.

966 Rokhlin were partially supported by the AFRL (USA) under the prime contract FA8650-10-

967 D-5210. M. Huang was supported by the Chinese Scholarship Council and the Beijing

968 Institute of Aeronautical Materials and M. J. S. Lowe was partially sponsored by the UK

969 Engineering and Physical Sciences Research Council (EPSRC). We also acknowledge the

970 software packages used: for Voronoi tessellation, Neper [27], meshing, Gmsh [47], and FE 971 solving on the GPU, Pogo [35].

\section{APPENDIX A: INNER PRODUCT COEFFICIENTS AND ELASTIC 973 SCATTERING FACTORS}

\section{1. Voigt velocities and inner product coefficients in the SOA model}

975 The homogenized Voigt elastic constants and velocities of a macroscopically isotropic 976 polycrystal of triclinic grains [39] are: 
977

$\left\langle c_{11}\right\rangle=\rho V_{0 L}^{2}=\frac{1}{5}\left(c_{11}+c_{22}+c_{33}\right)+\frac{2}{15}\left(c_{12}+c_{23}+c_{31}\right)+\frac{4}{15}\left(c_{44}+c_{55}+c_{66}\right)$

$\left\langle c_{44}\right\rangle=\rho V_{0 T}^{2}=\frac{1}{15}\left(c_{11}+c_{22}+c_{33}\right)-\frac{1}{15}\left(c_{12}+c_{23}+c_{31}\right)+\frac{1}{5}\left(c_{44}+c_{55}+c_{66}\right)$

978 where $c_{i j}$ are the elastic constants of the grains (total of 21 for the triclinic crystal).

979 The inner product coefficients $A_{L L}, B_{L L}, C_{L L}, A_{L T}, B_{L T}, C_{L T}, A_{T T}, B_{T T}, C_{T T}$ are given in Ref. [13] 980 using elastic covariances as:

$$
A_{L L}=\left\langle\delta c_{13} \delta c_{13}\right\rangle ; B_{L L}=-2\left\langle\delta c_{13} \delta c_{13}\right\rangle+4\left\langle\delta c_{15} \delta c_{15}\right\rangle+2\left\langle\delta c_{13} \delta c_{33}\right\rangle ;
$$

981

$C_{L L}=\left\langle\delta c_{33} \delta c_{33}\right\rangle+\left\langle\delta c_{13} \delta c_{13}\right\rangle-4\left\langle\delta c_{15} \delta c_{15}\right\rangle-2\left\langle\delta c_{13} \delta c_{33}\right\rangle$;

$$
A_{L T}=\left\langle\delta c_{14} \delta c_{14}\right\rangle+\left\langle\delta c_{15} \delta c_{15}\right\rangle ; B_{L T}=\left\langle\delta c_{15} \delta c_{15}\right\rangle-\left\langle\delta c_{14} \delta c_{14}\right\rangle+C_{L L} ; C_{L T}=-C_{L L}
$$

982 They can be further expressed analytically by quadratic dependence on elastic constants as

983 derived in Refs. [11, 45]; they were independently numerically checked in this work using 984 relevant covariances from Ref. [13]. The inner product coefficients $A_{T T}, B_{T T}, C_{T T}$ and $985 A_{T L}, B_{T L}, C_{T L}$ are given analytically through elastic constants in Ref. [44] (obtained with the 986 help of covariances from Refs. [11,45]). Also, the following equalities exist between the 987 remaining inner product coefficients:

$A_{L T}=A_{T L}, B_{L T}=B_{T L}, C_{L T}=C_{T L}$ and $C_{L L}=C_{T T}=-C_{T L}$.

\section{Elastic scattering factors in the FFA model}

990 The elastic scattering factors $Q_{M \rightarrow N}$ from the dispersion equations for the FFA model (Sec.

991 II.E, Eqs. 27, 29) are defined in Ref. [13] for polycrystals with triclinic grains through elastic 992 covariances. For the main incident longitudinal wave they are: 
993

$Q_{L \rightarrow L}=\frac{A_{L L}+B_{L L}+C_{L L}}{4\left\langle c_{11}\right\rangle^{2}}=\frac{\Delta_{5}}{4\left\langle c_{11}\right\rangle^{2}} ; \quad Q_{L \rightarrow T}=\frac{A_{L T}+\frac{1}{3} B_{L T}+\frac{1}{5} C_{L T}}{4\left\langle c_{11}\right\rangle\left\langle c_{44}\right\rangle}=\frac{\Delta_{7}}{4\left\langle c_{11}\right\rangle\left\langle c_{44}\right\rangle} ;$

994

$$
Q_{T \rightarrow T}=\frac{A_{T T}+B_{T T}+C_{T T}}{8\left\langle c_{44}\right\rangle^{2}}=\frac{\Delta_{6}^{T}}{8\left\langle c_{44}\right\rangle^{2}} ; Q_{T \rightarrow L}=\frac{A_{T L}+\frac{1}{3} B_{T L}+\frac{1}{5} C_{T L}}{8\left\langle c_{11}\right\rangle\left\langle c_{44}\right\rangle}=\frac{\Delta_{7}^{T}}{8\left\langle c_{11}\right\rangle\left\langle c_{44}\right\rangle}
$$

995 In Ref. [13] they were obtained numerically. For general anisotropy the analytical

996 expressions for inner products factors $\Delta_{5}, \Delta_{7}$ are given below:

997

$$
\begin{aligned}
\Delta_{5} & =\left\langle\delta c_{33} \delta c_{33}\right\rangle=\frac{1}{1575}\left(112 c_{11}^{2}+32 c_{12}^{2}+32 c_{13}^{2}+80 c_{14}^{2}+400 c_{15}^{2}+400 c_{16}^{2}+112 c_{22}^{2}+32 c_{23}^{2}\right. \\
& +400 c_{24}^{2}+80 c_{25}^{2}+400 c_{26}^{2}+112 c_{33}^{2}+400 c_{34}^{2}+400 c_{35}^{2}+80 c_{36}^{2}+128 c_{44}^{2}+320 c_{45}^{2}+320 c_{46}^{2} \\
& +128 c_{55}^{2}+320 c_{56}^{2}+128 c_{66}^{2}+16 c_{11} c_{12}+16 c_{11} c_{13}-16 c_{12} c_{13}-96 c_{11} c_{22}-64 c_{11} c_{23}+16 c_{12} c_{22} \\
& -16 c_{12} c_{23}-64 c_{13} c_{22}-16 c_{13} c_{23}+160 c_{14} c_{24}+160 c_{15} c_{25}+480 c_{16} c_{26}-96 c_{11} c_{33}-64 c_{12} c_{33} \\
& +16 c_{22} c_{23}+16 c_{13} c_{33}+160 c_{14} c_{34}+480 c_{15} c_{35}+160 c_{16} c_{36}-128 c_{11} c_{44}-96 c_{22} c_{33}-32 c_{12} c_{44} \\
& +16 c_{23} c_{33}-32 c_{13} c_{44}+480 c_{24} c_{34}+160 c_{25} c_{35}+320 c_{15} c_{46}+320 c_{16} c_{45}+160 c_{26} c_{36} \\
& +32 c_{11} c_{55}+32 c_{22} c_{44}-32 c_{12} c_{55}+128 c_{23} c_{44}+128 c_{13} c_{55}+320 c_{14} c_{56}+320 c_{25} c_{46} \\
& +320 c_{26} c_{45}+32 c_{11} c_{66}-128 c_{22} c_{55}+32 c_{33} c_{44}+128 c_{12} c_{66}-32 c_{23} c_{55}-32 c_{13} c_{66} \\
& +320 c_{24} c_{56}+320 c_{35} c_{46}+320 c_{36} c_{45}+32 c_{22} c_{66}+32 c_{33} c_{55}-32 c_{23} c_{66}+320 c_{34} c_{56} \\
& \left.-128 c_{33} c_{66}-64 c_{44} c_{55}-64 c_{44} c_{66}-64 c_{55} c_{66}\right)
\end{aligned}
$$

$$
\begin{aligned}
\Delta_{7} & =\frac{2}{3375}\left(41 c_{11}^{2}+71 c_{12}^{2}+71 c_{13}^{2}+195 c_{14}^{2}+195 c_{15}^{2}+195 c_{16}^{2}+41 c_{22}^{2}+71 c_{23}^{2}+195 c_{24}^{2}+195 c_{25}^{2}\right. \\
& +195 c_{26}^{2}+41 c_{33}^{2}+195 c_{34}^{2}+195 c_{35}^{2}+195 c_{36}^{2}+144 c_{44}^{2}+360 c_{45}^{2}+360 c_{46}^{2}+144 c_{55}^{2}+360 c_{56}^{2} \\
& +144 c_{66}^{2}-7 c_{11} c_{12}-7 c_{11} c_{13}-53 c_{12} c_{13}-23 c_{11} c_{22}-22 c_{11} c_{23}-7 c_{12} c_{22}-53 c_{12} c_{23}-22 c_{13} c_{22} \\
& -53 c_{13} c_{23}+30 c_{14} c_{24}+30 c_{15} c_{25}+30 c_{16} c_{26}-23 c_{11} c_{33}-22 c_{12} c_{33}-7 c_{22} c_{23}-7 c_{13} c_{33} \\
& +30 c_{14} c_{34}+30 c_{15} c_{35}+30 c_{16} c_{36}-24 c_{11} c_{44}-23 c_{22} c_{33}+24 c_{12} c_{44}-7 c_{23} c_{33}+24 c_{13} c_{44} \\
& +30 c_{24} c_{34}+30 c_{25} c_{35}+30 c_{26} c_{36}-24 c_{11} c_{55}-24 c_{22} c_{44}+24 c_{12} c_{55}+24 c_{23} c_{44}+24 c_{13} c_{55} \\
& -24 c_{11} c_{66}-24 c_{22} c_{55}-24 c_{33} c_{44}+24 c_{12} c_{66}+24 c_{23} c_{55}+24 c_{13} c_{66}-24 c_{22} c_{66}-24 c_{33} c_{55} \\
& \left.+24 c_{23} c_{66}-24 c_{33} c_{66}-72 c_{44} c_{55}-72 c_{44} c_{66}-72 c_{55} c_{66}\right)
\end{aligned}
$$

999 For the main incident transverse wave the $Q_{T \rightarrow T}$ is given in Ref. [44] and $\Delta_{7}^{T}=\Delta_{7}$, Eq. (A6) and $Q_{T \rightarrow L}=Q_{L \rightarrow T} / 2$. 
1003 The mass operator in the Born approximation is $m_{L}\left(k_{0 L}\right)=m_{L \rightarrow L}\left(k_{0 L}\right)+m_{L \rightarrow T}\left(k_{0 L}\right)$ and the

1004 Cauchy principal value of the integral in the first term $m_{L \rightarrow L}\left(k_{0 L}\right)$ can be rewritten as:

1005

$P . V \cdot \int_{0}^{\infty} \frac{\xi^{4}}{1-\xi^{2}} \int_{-1}^{+1}\left[\sum_{j=1}^{N} A_{j} a_{j}^{3} f_{L L}\left(k_{0 L}, a_{j}, x, \xi\right)\right] d x d \xi=$

$\sum_{j=1}^{N}\left\{A_{j} a_{j}^{3} P . V \cdot \int_{0}^{\infty} \frac{\xi^{4}}{\left(1-\xi^{2}\right)\left(1+k_{0 L}^{2} a_{j}^{2}+\xi^{2} k_{0 L}^{2} a_{j}^{2}\right)^{2}} \int_{-1}^{+1} \frac{A_{L L}+B_{L L} x^{2}+C_{L L} x^{4}}{\{1-D(\xi) x\}^{2}} d x d \xi\right\}$

1006 where $D(\xi)=\frac{2 \xi k_{0 L}^{2} a_{j}^{2}}{1+k_{0 L}^{2} a_{j}^{2}+\xi^{2} k_{0 L}^{2} a_{j}^{2}}$.

1007 The function $D(\xi)$ is introduced to simplify further approximations. At the Rayleigh limit,

1008 where $k_{0 L} a_{j}<<1$, the function $D(\xi)$ is upper bounded by the small value $k_{0 L} a_{j}$. The bound

1009 is easy to obtain since $D(\xi)$ is a function of $\xi$ with a single maximum at $\xi=1 /\left(k_{0 L} a_{j}\right)$ on the

1010 interval $0<\xi<\infty$ and approaches zero at the ends of the interval. As a result, we have

$1011 D(\xi)=2 \xi k_{0 L}^{2} a_{j}^{2} /\left(1+k_{0 L}^{2} a_{j}^{2}+\xi^{2} k_{0 L}^{2} a_{j}^{2}\right) \leq k_{0 L} a_{j}<<1$. While the inner integral can be evaluated

1012 exactly, it is beneficial to use a series expansion of the integrand over $x$ with the $D$ as a

1013 small parameter. The integral for the linear Dxterm vanishes and neglecting higher order

1014 terms we have:

1015

$\int_{-1}^{+1} \frac{A_{L L}+B_{L L} x^{2}+C_{L L} x^{4}}{[1-D x]^{2}} d x \approx 2 A_{L L}+\frac{2}{3} B_{L L}+\frac{2}{5} C_{L L}$

1016 which is a constant. The Cauchy principal value integral is rewritten as:

1017

$$
\sum_{j=1}^{N}\left\{A_{j} a_{j}^{3} P . V \cdot \int_{0}^{\infty} \frac{\xi^{4}}{\left(1-\xi^{2}\right)\left(1+k_{0 L}^{2} a_{j}^{2}+\xi^{2} k_{0 L}^{2} a_{j}^{2}\right)^{2}} d \xi\right\}=\frac{1}{2} \sum_{j=1}^{N}\left\{A_{j} a_{j}^{3} P . V \cdot \int_{-\infty}^{\infty} \frac{\xi^{4}}{\left(1-\xi^{2}\right)\left(1+k_{0 L}^{2} a_{j}^{2}+\xi^{2} k_{0 L}^{2} a_{j}^{2}\right)^{2}} d \xi\right\}
$$


1018 Note that the integrand decays as $1 /(\xi)^{2}$ at $\xi \rightarrow \infty$. After using contour integration and the 1019 residue theorem, it further simplifies to:

1020

$$
\frac{1}{2} \sum_{j=1}^{N}\left\{A_{j} a_{j}^{3} P . V \cdot \int_{-\infty}^{\infty} \frac{\xi^{4}}{\left(1-\xi^{2}\right)\left(1+k_{0 L}^{2} a_{j}^{2}+\xi^{2} k_{0 L}^{2} a_{j}^{2}\right)^{2}} d \xi\right\} \approx \frac{1}{2} \sum_{j=1}^{N}\left\{A_{j} a_{j}^{3}\left(-\frac{2 \pi}{4 a_{j}^{3} k_{0 L}^{3}}\right)\right\}=-\frac{\pi}{4 k_{0 L}^{3}}
$$

1021 Finally, by combining (B2) and (B4) one obtains for the double integral:

1022

$$
P . V . \int_{0}^{\infty} \frac{\xi^{4}}{1-\xi^{2}} \int_{-1}^{+1}\left[\sum_{j=1}^{N} A_{j} a_{j}^{3} f_{L L}\left(k_{0 L}, a_{j}, x, \xi\right)\right] d x d \xi=-\frac{\pi}{2 k_{0 L}^{3}}\left(A_{L L}+\frac{1}{3} B_{L L}+\frac{1}{5} C_{L L}\right)
$$

1023 Similarly for the LT term:

1024

$$
P . V \cdot \int_{0}^{\infty} \frac{\xi^{4}}{1-\xi^{2}} \int_{-1}^{+1}\left[\sum_{j=1}^{N} A_{j} a_{j}^{3} f_{L T}\left(k_{0 L}, a_{j}, x, \xi\right)\right] d x d \xi=-\frac{\pi}{2 k_{0 T}^{3}}\left(A_{L T}+\frac{1}{3} B_{L T}+\frac{1}{5} C_{L T}\right)
$$

1026 Therefore, the real part of the perturbed wavenumber at the Rayleigh limit is:

1027

$$
\operatorname{Re}[k]=k_{0 L}-\frac{\operatorname{Re}\left[m_{L}\left(k_{0 L}\right)\right]}{2 k_{0 L} V_{0 L}^{2}}=
$$

$$
k_{0 L}+\frac{k_{0 L}}{2 \rho^{2} V_{0 L}^{4}}\left(A_{L L}+\frac{1}{3} B_{L L}+\frac{1}{5} C_{L L}\right)+\frac{k_{0 L}}{2 \rho^{2} V_{0 L}^{2} V_{0 T}^{2}}\left(A_{L T}+\frac{1}{3} B_{L T}+\frac{1}{5} C_{L T}\right)
$$

1028 Eq. (B7) produces the real part of the perturbed wavenumber Eq. (17) in Sec. II. The 1029 derivation of the attenuation at the Rayleigh limit (related to the imaginary part in Eq. (17)) is 1030 similar to that performed in reference $[8,29]$ except for the accounting for the generalized TPC. The final result is given in Eq. (17). 


\section{APPENDIX C: DERIVATION OF THE STOCHASTIC ASYMPTOTES 1033 FOR PERTURBED WAVE NUMBER}

1034 The stochastic asymptote for the perturbed wave number is obtained from the SOA model at

1035 the high frequency limit $k_{0 L} a_{j} \gg>1$. As for the Rayleigh asymptote, the limit is evaluated

1036 after applying the Born approximation to the dispersion equation, Eq. (13). First we consider

1037 the real part of the mass operator terms. We pull out the $x$ independent term

$1038\left(1+k_{0 L}^{2} a_{j}^{2}+\xi^{2} k_{0 L}^{2} a_{j}^{2}\right)$ from the inner integrals in Eqs. (5-8) and observe that at high frequency

1039 the function $D(\xi)$ is upper bounded $D(\xi)=2 \xi k_{0 L}^{2} a_{j}^{2} /\left(1+k_{0 L}^{2} a_{j}^{2}+\xi^{2} k_{0 L}^{2} a_{j}^{2}\right)<2 \xi /\left(1+\xi^{2}\right)$ and for

$1040 k_{0 L} a_{j} \gg>1$ it is very close to this bound. Numerical and analytical evaluation show that the

1041 integrand $\left(A_{L L}+B_{L L} x^{2}+C_{L L} x^{4}\right) /[1-D(\xi) x]^{2}$ has a sharp peak at $\xi=1$ and $x \rightarrow 1$ due to the

1042 denominator. A similar integral was evaluated in [29] where such an integrand function peak

1043 was physically attributed to the domination of the forward wave scattering in the stochastic

1044 region; it is especially clear when the integral is represented in the spherical coordinates as in

1045 Ref. [13] (appendix A). The nominator is a smooth, relatively slowly changing function, and

1046 without loss of accuracy can be replaced by its value at $x=1$ and pulled out of the inner

1047 integral. The remaining inner integral can be easily taken and as a result the P.V. integral

1048 becomes:

1049

$$
\begin{aligned}
& P . V \cdot \int_{0}^{\infty} \frac{\xi^{4}}{\left(1-\xi^{2}\right)\left(1+k_{0 L}^{2} a_{j}^{2}+\xi^{2} k_{0 L}^{2} a_{j}^{2}\right)^{2}} \int_{-1}^{+1} \frac{A_{L L}+B_{L L} x^{2}+C_{L L} x^{4}}{[1-D(\xi) x]^{2}} d x d \xi \approx \\
& P . V \cdot \int_{0}^{\infty} \frac{\xi^{4}}{\left(1-\xi^{2}\right)\left(1+k_{0 L}^{2} a_{j}^{2}+\xi^{2} k_{0 L}^{2} a_{j}^{2}\right)^{2}} \frac{2\left(A_{L L}+B_{L L}+C_{L L}\right)}{1-D^{2}(\xi)} d \xi
\end{aligned}
$$

1050 The remaining Cauchy integral is explicitly solved by contour integration and the residue

1051 theorem. Under condition of $k_{0 L} a_{j}>1$ we have: 
1052

$P . V \cdot \int_{0}^{\infty} \frac{\xi^{4}}{\left(1-\xi^{2}\right)\left(1+k_{0 L}^{2} a_{j}^{2}+\xi^{2} k_{0 L}^{2} a_{j}^{2}\right)^{2}} \frac{2\left(A_{L L}+B_{L L}+C_{L L}\right)}{1-D^{2}(\xi)} d \xi \approx-\frac{5 \pi\left(A_{L L}+B_{L L}+C_{L L}\right)}{8 k_{0 L}^{3} a_{j}^{3}}$.

1053 Therefore, at high frequencies the real part of the integrals in Eq. (5) is further simplified as:

1054

$$
P . V \cdot \int_{0}^{\infty} \frac{\xi^{4}}{1-\xi^{2}} \int_{-1}^{+1}\left[\sum_{j=1}^{N} A_{j} a_{j}^{3} f_{L L}\left(k_{0 L}, a_{j}, x, \xi\right)\right] d x d \xi=\sum_{j=1}^{N}\left\{A_{j} a_{j}^{3}\left(-\frac{5 \pi\left(A_{L L}+B_{L L}+C_{L L}\right)}{8 k_{0 L}^{3} a_{j}^{3}}\right)\right\}=-\frac{5 \pi\left(A_{L L}+B_{L L}+C_{L L}\right)}{8 k_{0 L}^{3}}
$$

1055 From Eq. (C3), the real part of $m_{L \rightarrow L}\left(k_{0 L}\right)$ becomes:

1056

$$
\operatorname{Re}\left[m_{L \rightarrow L}\left(k_{0 L}\right)\right]=-\frac{5 k_{0 L}^{2}}{4 \rho^{2} V_{0 L}^{2}}\left(A_{L L}+B_{L L}+C_{L L}\right)
$$

Similarly, one can obtain the real part of $m_{L \rightarrow T}\left(k_{0 L}\right)$ as:

1058

$\operatorname{Re}\left[m_{L \rightarrow T}\left(k_{0 L}\right)\right]=-\frac{k_{0 L}^{2}}{\rho^{2} V_{0 T}^{2}} \frac{\left(A_{L T}+B_{L T}+C_{L T}\right)}{1-V_{0 L}^{2} / V_{0 T}^{2}}$

1059

Finally, the real part of the perturbed wavenumber at the high frequency limit is:

1060

$$
\operatorname{Re}[k]=k_{0 L}+\frac{5 k_{0 L}}{8 \rho^{2} V_{0 L}^{4}}\left(A_{L L}+B_{L L}+C_{L L}\right)+\frac{k_{0 L}}{2 \rho^{2} V_{0 L}^{2} V_{0 T}^{2}} \frac{\left(A_{L T}+B_{L T}+C_{L T}\right)}{1-V_{0 L}^{2} / V_{0 T}^{2}} .
$$

1061 This shows that the scattering into both longitudinal and transverse waves contributes to the 1062 stochastic limit for the real part of the perturbed wave number.

1063 The situation is different for the imaginary part of the mass operator in Eq. (5) that is related 1064 to the attenuation coefficient. It is well known $[8,29]$ that for longitudinal wave attenuation 1065 the L-L term dominates in the stochastic frequency range and the L-T term can be neglected.

1066 The derivation of the imaginary part of the wave number for the stochastic asymptote is 1067 similar to the simplifications for the inner integral in Eq. (C1), but at $D(\xi=1)$, and is 1068 analogous to derivation of the stochastic attenuation asymptote in Refs.[8,29] and these are 1069 not detailed here. 


\section{APPENDIX D: ELASTIC SCATTERING FACTORS IN THE} RAYLEIGH AND STOCHASTIC ASYMPTOTES

\section{Elastic scattering factors in the Rayleigh asymptotes for attenuation and} velocity

1074 In the Rayleigh frequency range the longitudinal velocity asymptote $V_{L}^{R}$ is independent of

1075 frequency and represents the quasi-static velocity limit $V_{L}^{q-\text {-st }}$ (Sec. II.C, Eq. (15)):

1076

$V_{L}^{R}=V_{L}^{q-s t}=V_{0 L}\left[1+2 Q_{L L}^{*}+2 Q_{L \rightarrow T}\right]^{-1}$

1077 The scattering coefficient $Q_{L \rightarrow T}$ is given by Eqs. (A4, A6), and the scattering factor $Q_{L L}^{*}$ is 1078 defined as:

1079

$$
Q_{L L}^{*}=\frac{\left(A_{L L}+B_{L L} / 3+C_{L L} / 5\right)}{4\left\langle c_{11}\right\rangle^{2}}=\frac{\Delta_{8}}{4\left\langle c_{11}\right\rangle^{2}} \cdot
$$

$$
\begin{aligned}
\Delta_{8} & =\frac{8}{3375}\left(11 c_{11}^{2}+16 c_{12}^{2}+16 c_{13}^{2}+45 c_{14}^{2}+45 c_{15}^{2}+45 c_{16}^{2}+11 c_{22}^{2}+16 c_{23}^{2}+45 c_{24}^{2}+45 c_{25}^{2}\right. \\
& +45 c_{26}^{2}+11 c_{33}^{2}+45 c_{34}^{2}+45 c_{35}^{2}+45 c_{36}^{2}+24 c_{44}^{2}+60 c_{45}^{2}+60 c_{46}^{2}+24 c_{55}^{2}+60 c_{56}^{2} \\
& +24 c_{66}^{2}+3 c_{11} c_{12}+3 c_{11} c_{13}-13 c_{12} c_{13}-8 c_{11} c_{22}-12 c_{11} c_{23}+3 c_{12} c_{22}-13 c_{12} c_{23}-12 c_{13} c_{22} \\
& -13 c_{13} c_{23}+30 c_{14} c_{24}+30 c_{15} c_{25}+30 c_{16} c_{26}-8 c_{11} c_{33}-12 c_{12} c_{33}+3 c_{22} c_{23}+3 c_{13} c_{33} \\
& +30 c_{14} c_{34}+30 c_{15} c_{35}+30 c_{16} c_{36}-4 c_{11} c_{44}-8 c_{22} c_{33}+4 c_{12} c_{44}+3 c_{23} c_{33}+4 c_{13} c_{44} \\
& +30 c_{24} c_{34}+30 c_{25} c_{35}+30 c_{26} c_{36}-4 c_{11} c_{55}-4 c_{22} c_{44}+4 c_{12} c_{55}+4 c_{23} c_{44}+4 c_{13} c_{55} \\
& -4 c_{11} c_{66}-4 c_{22} c_{55}-4 c_{33} c_{44}+4 c_{12} c_{66}+4 c_{23} c_{55}+4 c_{13} c_{66}-4 c_{22} c_{66}-4 c_{33} c_{55} \\
& \left.+4 c_{23} c_{66}-4 c_{33} c_{66}-12 c_{44} c_{55}-12 c_{44} c_{66}-12 c_{55} c_{66}\right)
\end{aligned}
$$

1082 The Rayleigh asymptote for the attenuation coefficient $\alpha_{L}^{R}$, Eq. (16), depends on the same 1083 elastic scattering factors $Q_{L L}^{*}, Q_{L \rightarrow T}$.

\section{Elastic scattering factors in the stochastic asymptotes for attenuation and velocities}

1085 The stochastic velocity asymptote $V_{L}^{S}$ is also independent of frequency (Sec. II.D, Eq. (21)): 
$V_{L}^{S}=V_{0 L}\left[1+\frac{5 Q_{L \rightarrow L}}{2}-\frac{\left(A_{L T}+B_{L T}+C_{L T}\right)}{2\left\langle c_{11}\right\rangle\left(\left\langle c_{11}\right\rangle-\left\langle c_{44}\right\rangle\right)}\right]^{-1}$

1087 The stochastic velocity limits depend on both the longitudinal-to-longitudinal (second term)

1088 and the longitudinal-to-transverse (third term) wave scattering. The scattering factor $Q_{L \rightarrow L}$ is

1089 given by Eqs. (A4, A5), and the scattering factor $\Delta_{9}=\left(A_{L T}+B_{L T}+C_{L T}\right)$ is analytically

1090 represented as:

1091

$$
\begin{aligned}
\Delta_{9} & =\frac{2}{315}\left(5 c_{11}^{2}+3 c_{12}^{2}+33_{13}^{2}+7 c_{14}^{2}+23 c_{15}^{2}+23 c_{16}^{2}+5 c_{22}^{2}+3 c_{23}^{2}+23 c_{24}^{2}+7 c_{25}^{2}\right. \\
& +23 c_{26}^{2}+5 c_{33}^{2}+23 c_{34}^{2}+23 c_{35}^{2}+7 c_{36}^{2}+12 c_{44}^{2}+28 c_{45}^{2}+28 c_{46}^{2}+12 c_{55}^{2}+28 c_{56}^{2} \\
& +12 c_{66}^{2}-c_{11} c_{12}-c_{11} c_{13}-c_{12} c_{13}-3 c_{11} c_{22}-2 c_{11} c_{23}-c_{12} c_{22}-c_{12} c_{23}-2 c_{13} c_{22} \\
& -c_{13} c_{23}+2 c_{14} c_{24}+2 c_{15} c_{25}+6 c_{16} c_{26}-3 c_{11} c_{33}-2 c_{12} c_{33}-c_{22} c_{23}-c_{13} c_{33} \\
& +2 c_{14} c_{34}+6 c_{15} c_{35}+2 c_{16} c_{36}-4 c_{11} c_{44}-3 c_{22} c_{33}-2 c_{12} c_{44}-c_{23} c_{33}-2 c_{13} c_{44} \\
& +6 c_{24} c_{34}+2 c_{25} c_{35}+2 c_{26} c_{36}-2 c_{11} c_{55}-2 c_{22} c_{44}-2 c_{12} c_{55}+12 c_{23} c_{44}+12 c_{13} c_{55} \\
& -2 c_{11} c_{66}-4 c_{22} c_{55}-2 c_{33} c_{44}+12 c_{12} c_{66}-2 c_{23} c_{55}-2 c_{13} c_{66}-2 c_{22} c_{66}-2 c_{33} c_{55} \\
& -2 c_{23} c_{66}-4 c_{33} c_{66}-4 c_{44} c_{55}-4 c_{44} c_{66}-4 c_{55} c_{66}+4 c_{16} c_{45}+4 c_{26} c_{45}+28 c_{36} c_{45} \\
& \left.+4 c_{15} c_{46}+28 c_{25} c_{46}+4 c_{35} c_{46}+28 c_{14} c_{56}+4 c_{24} c_{56}+4 c_{34} c_{56}\right)
\end{aligned}
$$

1092 The stochastic asymptote for the attenuation (Eq. (22)) is

1093

$$
\alpha_{L}^{S}=k_{0 L}^{2} Q_{L \rightarrow L} \sum_{j=1}^{N} A_{j} a_{j}
$$

1094 It depends on frequency and through the generalized TPC on grain size. The dependence on

1095 only one longitudinal scattering factor $Q_{L \rightarrow L}$ indicates the well-known fact that only

1096 longitudinal-to-longitudinal wave scattering is accounted for in the stochastic attenuation

1097 asymptote. 


\section{References:}

[1] Topics in Applied Physics. Imaging of Complex Media with Acoustic and Seismic Waves, edited by M. Fink, W. A. Kuperman, J.-P. Montagner, and A. Tourin (Springer-Verlag, Berlin, Germany, 2002),Vol. 84, pp. 1-336.

[2] H. Sato, M. C. Fehler, and T. Maeda, Seismic wave propagation and scattering in the heterogeneous earth, 2nd Ed Spring Verlag, Berlin 2012.

[3] R. B. Thompson and F.J. Margetan "Use of elastodynamic theories in the stochastic description of the effects of microstructure on ultrasonic flaw and noise signals" Wave Motion 36, 347-365 (2002)

[4] F. E. Stanke and G. S. Kino, "A unified theory for elastic wave propagation in polycrystalline materials," J. Acoust. Soc. Am., 75(3), 665-681 (1984).

[5] R. L. Weaver, "Diffusivity of ultrasound in polycrystals," J. Mech. Phys. Solids, 38(1), 55-86 (1990).

[6] S. Hirsekorn, "The scattering of ultrasonic waves by polycrystals," J. Acoust. Soc. Am. 72(3), 1021-1031 (1982).

[7] J. A. Turner, "Elastic wave propagation and scattering in heterogeneous, anisotropic media: Textured polycrystalline materials," J. Acoust. Soc. Am.106 (2), 541-552 (1999).

[8] J. Li and S. I. Rokhlin, "Propagation and scattering of ultrasonic waves in polycrystals with arbitrary crystallite and macroscopic texture symmetries," Wave Motion, 58, 145164(2015).

[9] A. L. Pilchak, J. Li, and S. I. Rokhlin, "Quantitative Comparison of Microtexture in Near-Alpha Titanium Measured by Ultrasonic Scattering and Electron Backscatter 
Diffraction," Metall. Mater. Trans. A45 (10), 4679-4697 (2014).

[10] A. P. Arguelles, C. M. Kube, P. Hu, and J. A. Turner, "Mode-converted ultrasonic scattering in polycrystals with elongated grains," J. Acoust. Soc. Am. 140(3), 15701580 (2016).

[11] C. M. Kube and J. A. Turner, "Acoustic attenuation coefficients for polycrystalline materials containing crystallites of any symmetry class," J Acoust Soc Am. 137(6), El476-82 (2015).

[12] J. Li and S. I. Rokhlin, "Elastic wave scattering in random anisotropic solids," Int. J. Solids Struct. 78-79, 110-124 (2016).

[13] S. I. Rokhlin, J. Li, and G. Sha, "Far-field scattering model for wave propagation in random media,” J. Acoust. Soc. Am.137 (5), 2655-2669 ( 2015).

[14] S. Ahmed and R. B. Thompson, "Attenuation of ultrasonic waves in cubic metals having elongated, oriented grains," Nondestruct. Test. Eval. 8-9, 525-531(1992).

[15] M. Calvet and L. Margerin, "Impact of grain shape on seismic attenuation and phase velocity in cubic polycrystalline materials," Wave Motion, 65, 29-43 (2016).

[16] S. Hirsekorn, "The scattering of ultrasonic waves in polycrystalline materials with texture," J. Acoust. Soc. Am. 77 (3), 832-843 (1985).

[17] C. M. Kube and J. A. Turner, "Stress-dependent ultrasonic scattering in polycrystalline materials," J. Acoust. Soc. Am. 139(2), 811-824 (2016).

[18] J.-Y. Kim and S. I. Rokhlin, "Determination of elastic constants of generally anisotropic inclined lamellar structure using line-focus acoustic microscopy.," $J$. Acoust. Soc. Am., 126 (6), 2998-3007(2009). 
[19] M. Calvet and L. Margerin, "Velocity and attenuation of scalar and elastic waves in random media: A spectral function approach,” J. Acoust. Soc. Am.131 (3), 1843-1862, (2012).

[20] L. Yang, O. I. Lobkis, and S. I. Rokhlin, "An integrated model for ultrasonic wave propagation and scattering in a polycrystalline medium with elongated hexagonal grains," Wave Motion 49(5), 544-560(2012).

[21] A. Van Pamel, G. Sha, S. I. Rokhlin, and M. J. S. Lowe, "Finite-element modelling of elastic wave propagation and scattering within heterogeneous media," Proc. R. Soc. A, 473, $20160738,1-21(2017)$.

[22] M. Ryzy, T. Grabec, P. Sedlak, and I. A. Veres, "Influence of grain morphology on ultrasonic wave attenuation in polycrystalline media with statistically equiaxed grains," J. Acoust. Soc. Am.143 (1), 219-229 (2018).

[23] A. Van Pamel, G. Sha, M. J. S. Lowe, and S. I. Rokhlin, "Numerical and analytic modelling of elastodynamic scattering within polycrystalline materials," J. Acoust. Soc. Am. 143(4), 2394-2408 (2018).

[24] P.E. Lhuiller, B. Chassignole, M. Oudaa, S.O. Kerherve, F.Rupin and T.F. Fouquet "Investigation of the ultrasonic attenuation in anisotropic weld materials with finite element modeling and grain-scale material description," Ultrasonics 78, 40-50 (2017).

[25] A. Van Pamel, C. R. Brett, P. Huthwaite, and M. J. S. Lowe, "Finite element modelling of elastic wave scattering within a polycrystalline material in two and three dimensions," J. Acoust. Soc. Am. 138 (4), 2326-2336 (2015).

[26] Y. Liu, A. Van Pamel, P. B. Nagy, and P. Cawley "Investigation of ultrasonic backscatter using three-dimensional finite element simulations” J. Acoust. Soc. Am. 
145 (3), 1584-1595 (2019).

[27] R. Quey, P. R. Dawson, and F. Barbe, "Large-scale 3D random polycrystals for the finite element method: Generation, meshing and remeshing," Comput. Methods Appl. Mech. Eng. 200 (17-20), 729-1745 (2011).

[28] R. B. Thompson, "Elastic-wave propagation in random polycrystals: Fundamentals and application to nondestructive evaluation," in Topics in Applied Physics. Imaging of Complex Media with Acoustic and Seismic Waves, edited by M. Fink, W. A. Kuperman, J.-P. Montagner, and A. Tourin (Springer-Verlag, Berlin, Germany, 2002), Vol. 84,pp. 233-257.

[29] L. Yang, O. I. Lobkis, and S. I. Rokhlin, "Shape effect of elongated grains on ultrasonic attenuation in polycrystalline materials," Ultrasonics 51(6), 697-708 (2011).

[30] C. S. Man, R. Paroni, Y. Xiang, and E. A. Kenik, "On the geometric autocorrelation function of polycrystalline materials," J. Comput. Appl. Math.190, (1-2), 200-210 (2006).

[31] G. Sha, “Analytical attenuation and scattering models for polycrystals with uniform equiaxed grains," J. Acoust. Soc. Am. 143 (5), EL347-EL353 (2018).

[32] F. E. Stanke, "Spatial autocorrelation functions for calculations of effective propagation constants in polycrystalline materials," J. Acoust. Soc. Am. 80 (5), 14791485 (1986).

[33] K. Sobczyk and D. J. Kirkner, "Material media microstructure: modeling issues," in Stochastic Modeling of Microstructures, Boston, MA: Birkhäuser Boston, 2001, pp. 149-202. 
[34] A. Van Pamel, P. B. Nagy, and M. J. S. Lowe, "On the dimensionality of elastic wave scattering within heterogeneous media," J. Acoust. Soc. Am. 140(6), 4360-4366 (2016).

[35] P. Huthwaite, "Accelerated finite element elastodynamic simulations using the GPU," J. Comput. Phys. 257, 687-707 (2014).

[36] ASTM E112-12, "Standard Test Methods for Determining Average Grain Size," ASTM International 2012.

[37] C. M. Kube and A. P. Arguelles, "Bounds and self-consistent estimates of the elastic constants of polycrystals," Comput. Geosci. 95, 118-122 (2016).

[38] J. M. Brown, "Determination of Hashin-Shtrikman bounds on the isotropic effective elastic moduli of polycrystals of any symmetry," Comput. Geosci. 80, 95-99 (2015).

[39] R. Hill, "The elastic behaviour of a crystalline aggregate," Proc. Phys. Soc. Sect. A 65 (5) 349-354 (1952).

[40] M. Lobos and T. Böhlke, "Materials design for the anisotropic linear elastic properties of textured cubic crystal aggregates using zeroth-, first- and second-order bounds," Int. J. Mech. Mater. Des. 11 (1), 59-78( 2015).

[41] M. R. Murshed and S. I. Ranganathan, "Scaling laws in elastic polycrystals with individual grains belonging to any crystal class," Acta Mech. 228(4), 1525-1539 ( 2017).

[42] S. I. Ranganathan and M. Ostoja-Starzewski, "Universal elastic anisotropy index," Phys. Rev. Lett. 101(5), 055504, 3-6, (2008).

[43] C. M. Kube, "Elastic anisotropy of crystals," AIP Adv. 6, 095209 (2016). 
[44] G. Sha and S. I. Rokhlin, "Universal scaling of transverse wave attenuation in polycrystals," Ultrasonics 88, 84-96 (2018).

[45] C. M. Kube, "Erratum: Acoustic attenuation coefficients for polycrystalline materials containing crystallites of any symmetry class (J Acoust Soc Am. 137(6), El476-82 (2015)),” J. Acoust. Soc. Am. 138, 976 (2015).)

[46] O. I. Lobkis, L. Yang, J. Li and S. I. Rokhlin,"Ultrasonic backscattering in polycrystals with elongated single phase and duplex microstructures" Ultrasonics 52(6), 694-705 (2012).

[47] C. Geuzaine and J.-F. Remacle, "Gmsh: A 3-D finite element mesh generator with built-in pre- and post-processing facilities," Int. J. Numer. Methods Eng. 79(11), 13091331 (2009). 
Table I. Elastic constants $c_{i j}$ (in GPa), density $\rho$ (in $\mathrm{g} / \mathrm{cm} 3$ ), elastic scattering factors $Q_{M \rightarrow N}$ and universal anisotropy factor $A^{U}$ of triclinic symmetry copper sulfate pentahydrate $(\mathrm{CSP})[13] ; Q_{L \rightarrow T}=2 Q_{T \rightarrow L}$.

\begin{tabular}{lccccccccc}
\hline \hline & $c_{11}$ & $c_{12}$ & $c_{13}$ & $c_{14}$ & $c_{15}$ & $c_{16}$ & $c_{22}$ & $c_{23}$ & $c_{24}$ \\
\hline $\mathrm{CSP}$ & 56.5 & 26.5 & 32.1 & -3.3 & -0.8 & -3.9 & 43.3 & 34.7 & -0.7 \\
\hline \hline $\mathrm{CSP}$ & $c_{25}$ & $c_{26}$ & $c_{33}$ & $c_{34}$ & $c_{35}$ & $c_{36}$ & $c_{44}$ & $c_{45}$ & $c_{46}$ \\
\hline \hline & -2.1 & 2.0 & 56.9 & -4.4 & -2.1 & -1.6 & 17.3 & 0.9 & 0.3 \\
\hline $\mathrm{CSP}$ & $c_{55}$ & $c_{56}$ & $c_{66}$ & $\rho$ & $Q_{L \rightarrow L}$ & $Q_{L \rightarrow T}$ & $Q_{T \rightarrow T}$ & $A^{U}$ \\
\hline \hline
\end{tabular}


Table II. Details of spatial models for wave scattering modelling at different frequencies.

\begin{tabular}{|c|c|c|c|c|c|c|c|c|}
\hline \multirow{2}{*}{$\begin{array}{l}\text { Model } \\
\text { name }\end{array}$} & \multicolumn{3}{|c|}{$\begin{array}{l}\text { Model Size } \\
(\mathrm{mm})\end{array}$} & \multirow{2}{*}{$\begin{array}{l}\text { Mean } \\
\text { Grain } \\
\text { Size }(\mathrm{mm})\end{array}$} & \multirow{2}{*}{$\begin{array}{l}\text { Element } \\
\text { Size } \\
(\mathrm{mm})\end{array}$} & \multirow{2}{*}{ DOF } & \multirow{2}{*}{$\begin{array}{l}\text { Centre } \\
\text { frequency } \\
(\mathrm{MHz})\end{array}$} & \multirow{2}{*}{ Realizations } \\
\hline & $X$ & $Y$ & $Z$ & & & & & \\
\hline N115200 & 12 & 12 & 100 & & 0.050 & $3.49 \times 10^{8}$ & 1,2 & \\
\hline N11520 & 12 & 12 & 10 & 0.5 & 0.025 & $2.78 \times 10^{8}$ & $3,5,7,9$ & 15 \\
\hline N16000 & 20 & 20 & 5 & & 0.020 & $7.55 \times 10^{8}$ & 18 & \\
\hline
\end{tabular}


Table III. Material properties of homogeneous isotropic steel.

\begin{tabular}{lllll}
\hline \hline$E(\mathrm{GPa})$ & $v$ & $\rho\left(\mathrm{kg} / \mathrm{m}^{3}\right)$ & Analytical $V_{L}^{S t}(\mathrm{~m} / \mathrm{s})$ & $\mathrm{FEM} V_{L}^{S t}(\mathrm{~m} / \mathrm{s})$ \\
\hline 210 & 0.3 & 8000 & 5944.4544 & 5944.0796 \\
\hline \hline
\end{tabular}


Table IV. Coefficients of Generalized TPC series shown in Figure 4.

\begin{tabular}{ccc}
\hline TPC Series & $A_{i}$ & $a_{i}$ \\
\hline Term 1 & -2922.66 & 0.115726 \\
Term 2 & -10.6181 & 0.172221 \\
Term 3 & 3914.370 & 0.110790 \\
Term 4 & -3305.68 & 0.103201 \\
Term 5 & 696.919 & 0.0910265 \\
Term 6 & 54.3582 & 0.152263 \\
Term 7 & -42.4732 & 0.0723540 \\
Term 8 & 1616.78 & 0.110790 \\
\hline \hline
\end{tabular}


Table V. Comparison of the discrepancy (RMSD) between the analytical and FE models for different material systems (FEM is taken as the reference for the RMSD). The universal anisotropy factor $A^{U}$, scattering factors $Q_{L \rightarrow M}$ and the attenuation, predicted by the SOA model at certain frequencies, are also given.

\begin{tabular}{|c|c|c|c|c|c|c|c|}
\hline \multirow{2}{*}{$\begin{array}{l}\text { Material } \\
\mathrm{s}\end{array}$} & \multirow{2}{*}{$\begin{array}{l}\text { Universal } \\
\text { Anisotrop } \\
\text { y } A^{U}\end{array}$} & \multirow[t]{2}{*}{$Q_{L \rightarrow L}$} & \multirow{2}{*}{$Q_{L \rightarrow T}$} & \multicolumn{2}{|c|}{$\begin{array}{l}\text { RMSD } \\
\text { (with respect to } \\
\text { the FEM) }\end{array}$} & \multicolumn{2}{|c|}{ Attenuation (SOA) } \\
\hline & & & & SOA & FFA & $2 k_{L} a_{l}=1$ & $2 k_{L} a_{l}=20$ \\
\hline $\mathrm{Al}$ & 0.054 & $7.80 \times 10^{-5}$ & $3.34 \times 10^{-4}$ & $\begin{array}{l}7.35 \\
\%\end{array}$ & $5.81 \%$ & $1.40 \times 10^{-4}$ & $1.27 \times 10^{-2}$ \\
\hline $\mathrm{A}=1.5$ & 0.21 & $3.88 \times 10^{-4}$ & $1.43 \times 10^{-3}$ & $\begin{array}{l}7.09 \\
\%\end{array}$ & $5.38 \%$ & $5.03 \times 10^{-4}$ & $6.41 \times 10^{-2}$ \\
\hline $\mathrm{A}=1.8$ & 0.45 & $7.60 \times 10^{-4}$ & $2.79 \times 10^{-3}$ & $\begin{array}{l}7.60 \\
\%\end{array}$ & $7.52 \%$ & $9.52 \times 10^{-4}$ & $1.21 \times 10^{-1}$ \\
\hline $\mathrm{A}=2.4$ & 1.03 & $1.49 \times 10^{-3}$ & $5.48 \times 10^{-3}$ & $\begin{array}{l}12.6 \\
\%\end{array}$ & $\begin{array}{l}13.75 \\
\%\end{array}$ & $1.99 \times 10^{-3}$ & $2.41 \times 10^{-1}$ \\
\hline Inconel & 1.42 & $2.26 \times 10^{-3}$ & $7.59 \times 10^{-3}$ & $\begin{array}{l}14.6 \\
\%\end{array}$ & $15.4 \%$ & $2.54 \times 10^{-3}$ & $3.74 \times 10^{-1}$ \\
\hline Copper & 1.75 & $1.76 \times 10^{-3}$ & $7.21 \times 10^{-3}$ & $\begin{array}{l}14.1 \\
\%\end{array}$ & $15.0 \%$ & $3.09 \times 10^{-3}$ & $2.93 \times 10^{-1}$ \\
\hline CSP & 0.948 & $3.23 \times 10^{-3}$ & $7.19 \times 10^{-3}$ & $\begin{array}{l}9.44 \\
\%\end{array}$ & $7.77 \%$ & $3.76 \times 10^{-3}$ & $5.03 \times 10^{-1}$ \\
\hline
\end{tabular}


Table VI. Static longitudinal velocity $V_{L}^{S t}=\sqrt{C_{11} / \rho}$ (in $\mathrm{km} / \mathrm{s}$ ) and elastic constants $C_{11}, C_{44}$ (in GPa) for the homogenized CSP polycrystal using different homogenization models and the results of this work. Eq. (15) relates to the Rayleigh limit of $V_{L}^{S t}$ and Eq. (18) of $V_{T}^{S t}$.

\begin{tabular}{|c|c|c|c|c|c|c|c|c|c|c|}
\hline Limit & $\begin{array}{l}\text { Reus } \\
\mathrm{s}\end{array}$ & $\begin{array}{l}\mathrm{H}-\mathrm{S} \\
\mathrm{L}\end{array}$ & Hill & SC & $\begin{array}{l}\mathrm{SOA} \\
\left(2 k_{L} a_{l}=0.01\right.\end{array}$ & $\begin{array}{l}\text { Eq.(15) } \\
\text { Eq.(18) }\end{array}$ & $\begin{array}{l}\text { FFA with } \\
\text { correctio } \\
\text { n }\end{array}$ & FEM & $\begin{array}{l}\mathrm{H}-\mathrm{S} \\
\mathrm{U}\end{array}$ & $\begin{array}{l}\text { Voig } \\
\mathrm{t}\end{array}$ \\
\hline$C_{11}$ & 50.01 & $\begin{array}{l}51.8 \\
9\end{array}$ & $\begin{array}{l}52.1 \\
6\end{array}$ & $\begin{array}{l}52.3 \\
6\end{array}$ & 52.38 & 52.45 & 52.45 & $\begin{array}{l}52.4 \\
7\end{array}$ & $\begin{array}{l}52.7 \\
1\end{array}$ & $\begin{array}{l}54.3 \\
1\end{array}$ \\
\hline$V_{L}^{S t}=\sqrt{C_{11} / \rho}$ & 4.677 & $\begin{array}{l}4.76 \\
4\end{array}$ & $\begin{array}{l}4.77 \\
7\end{array}$ & $\begin{array}{l}4.78 \\
6\end{array}$ & 4.787 & 4.790 & 4.790 & $\begin{array}{l}4.79 \\
1\end{array}$ & $\begin{array}{l}4.80 \\
2\end{array}$ & $\begin{array}{l}4.87 \\
4\end{array}$ \\
\hline$C_{44}$ & 10.28 & $\begin{array}{l}11.0 \\
9\end{array}$ & $\begin{array}{l}11.2 \\
1\end{array}$ & $\begin{array}{l}11.2 \\
9\end{array}$ & 11.33 & 11.37 & 11.37 & - & $\begin{array}{l}11.4 \\
4\end{array}$ & $\begin{array}{l}12.1 \\
3\end{array}$ \\
\hline
\end{tabular}


Table VII. Anisotropy factors calculated for all materials systems used in this and previous studies $[21,23]$ using definitions from $[42,43]$.

\begin{tabular}{cccc}
\hline \hline & & & \\
Materials & Universal Anisotropy & Equivalent Anisotropy & Anisotropy factor \\
& $A^{U}[42]$ & $A^{E}[42]$ & $A^{L}[43]$ \\
Al[20] & 0.054 & 1.24 & 0.0239 \\
$\mathrm{~A}=1.5[23]$ & 0.21 & 1.52 & 0.0928 \\
$\mathrm{~A}=1.8[23]$ & 0.45 & 1.83 & 0.192 \\
$\mathrm{~A}=2.4[23]$ & 1.03 & 2.45 & 0.418 \\
Inconel [21] & 1.42 & 2.83 & 0.559 \\
Copper[23] & 1.75 & 3.14 & 0.670 \\
CSP Table 1 and & & & 0.372 \\
\hline 13$]$ & 0.948 & 2.37 & \\
\hline \hline
\end{tabular}


Table VIII. Poisson's ratio, $\frac{V_{0 L}^{3}}{V_{0 T}^{3}}, \sum_{j=1}^{N} A_{j} a_{j}^{3}$ and $\sum_{j=1}^{N} A_{j} a_{j}$ for CSP, Copper and Inconel, and numerical values for correlation length $a_{C L}$ and effective grain volume $V_{g}^{\text {eff }}$ for the actual FEM TPC.

\begin{tabular}{cccc}
\hline \hline Materials & CSP & Copper & Inconel \\
\hline Poisson's ratio & 0.3563 & 0.3275 & 0.2733 \\
$\frac{V_{0 L}^{3}}{V_{0 T}^{3}}$ & 9.479 & 7.699 & 5.738 \\
$V_{g}^{R}=\sum_{j=1}^{N} A_{j} a_{j}^{3}$ & 0.005766 & 0.007385 & 0.007385 \\
$V_{g}^{e f f}=8 \pi \sum_{j=1}^{N} A_{j} a_{j}^{3}$ & 0.1449 & & \\
$a_{C L}=\sum_{j=1}^{N} A_{j} a_{j}$ & 0.2309 & 0.1856 & 0.1856 \\
\end{tabular}

Numerical from FE material

Correlation length, $\mathrm{mm}$

$$
a_{C L}=\int_{0}^{\infty} w(r) d r
$$

Numerical from FE material

Effective volume, $\mathrm{mm}^{3}$

0.149

$$
V_{g}^{e f f}=\int_{V} w(r) d V
$$




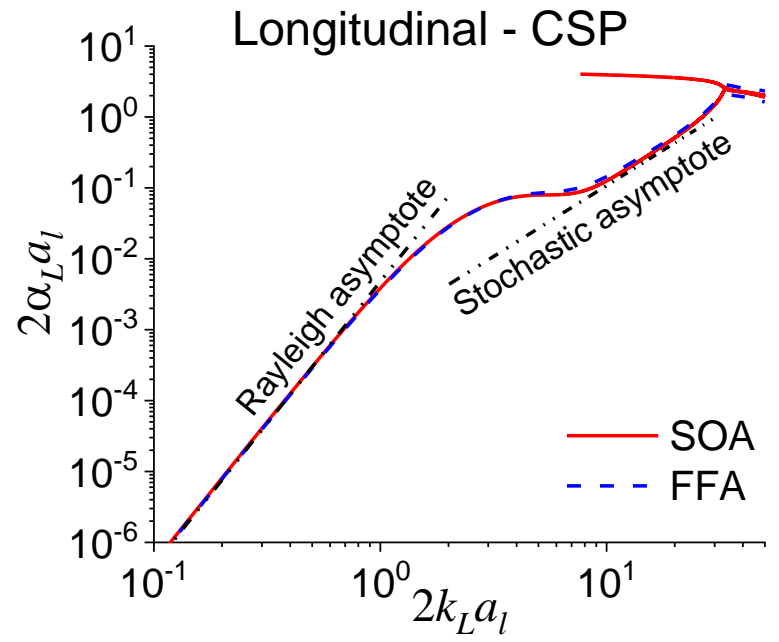

(a)

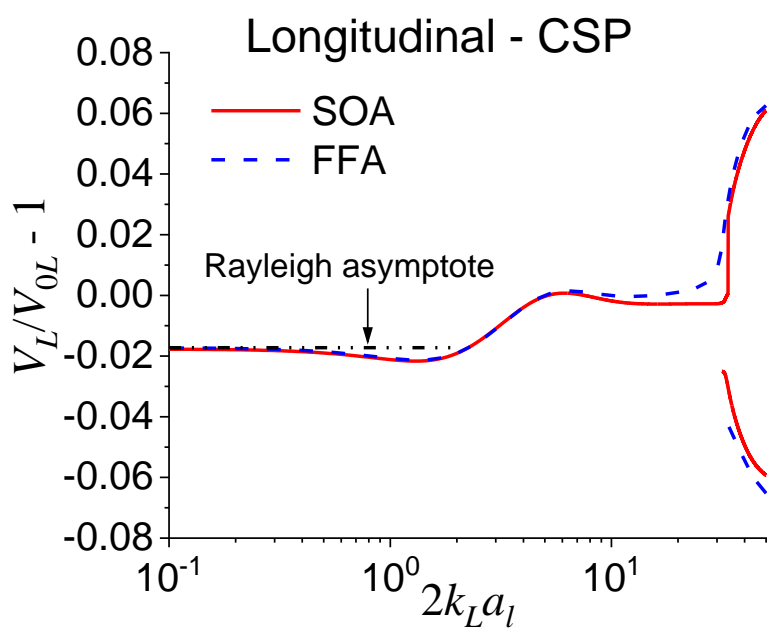

(c)

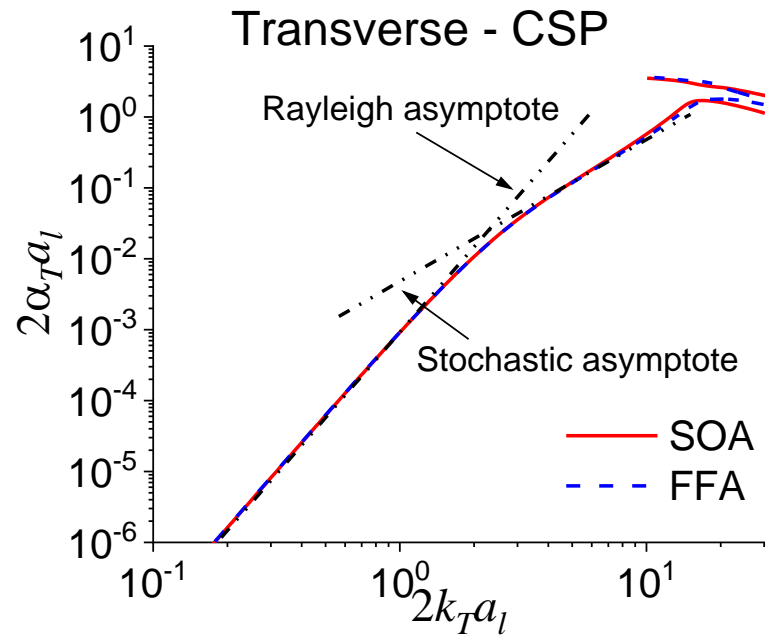

(b)

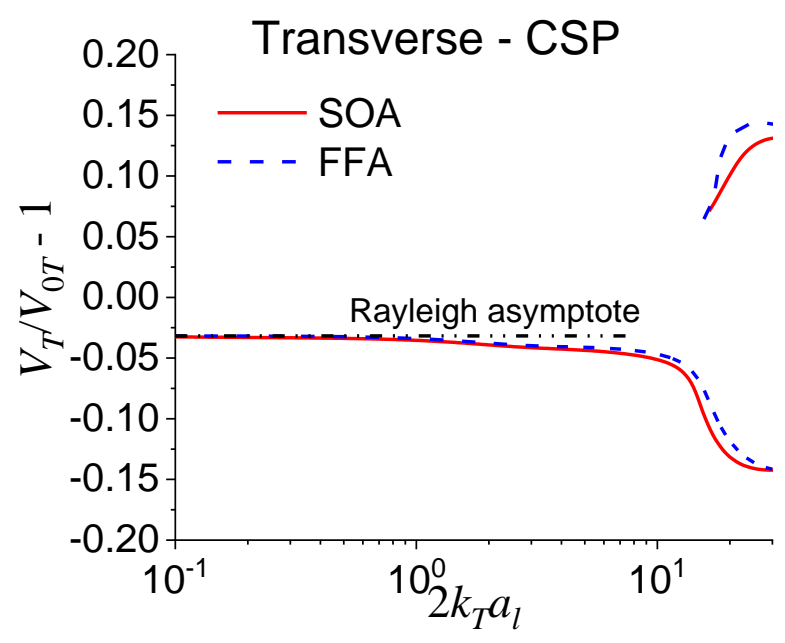

(d)

Figure 1. (Color online) Comparison of the SOA and FFA models in all frequency regimes for the normalized attenuation $(a, b)$ and velocity $(c, d)$ of longitudinal $(a, c)$ and transverse (b,d) waves. The calculations are for the CSP polycrystal with equiaxed grains and the generalized TPC function. The two roots of the dispersion equation from the SOA and FFA models are shown. For the FFA model the velocities are with the correction given in Eqs. $(28,30)$. 


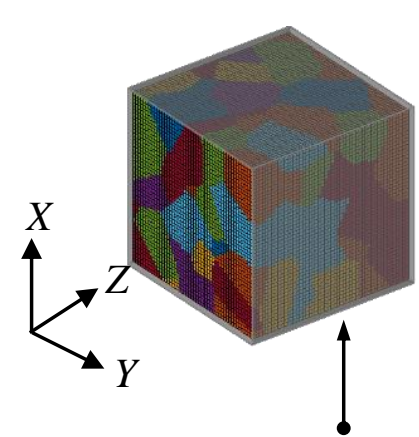

Symmetry boundary conditions

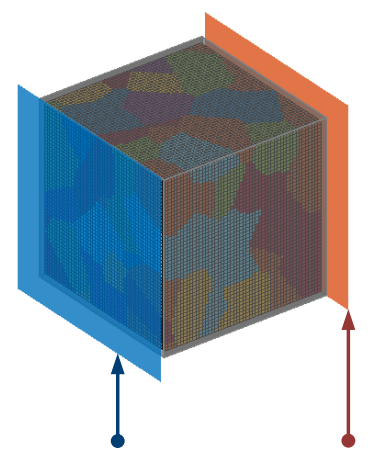

Transmitting Receiving
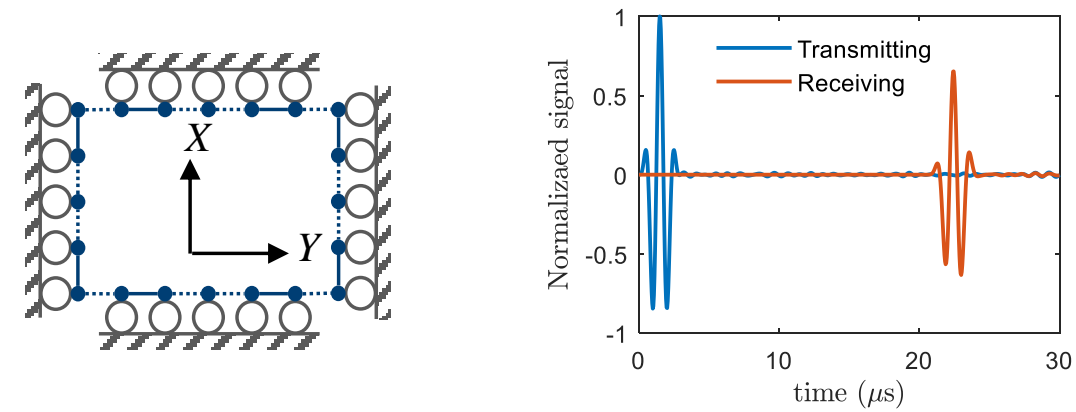

Figure 2. (Color online) Three-dimensional cuboid FE domain, showing boundary conditions, transmitting (loading) and receiving planes, and typical example coherent signal. 


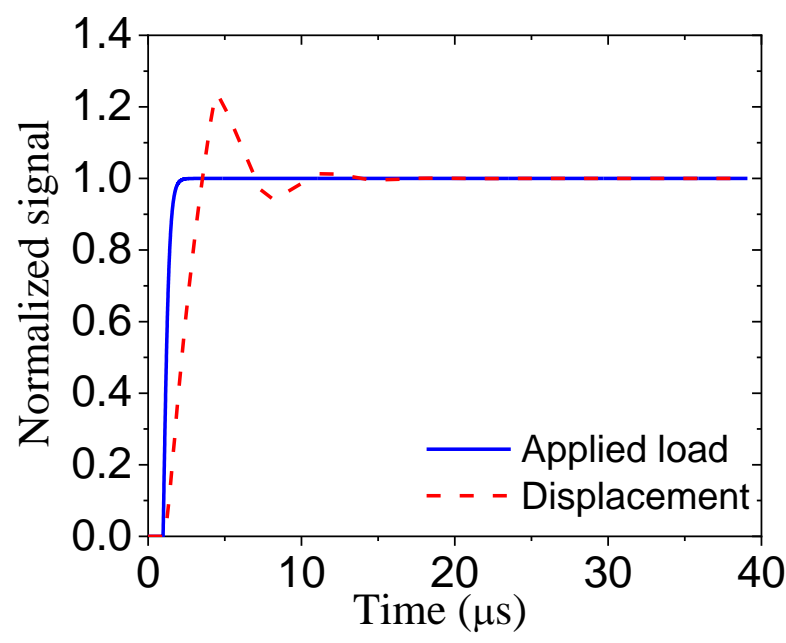

Figure 3. (Color online) A typical example of loading and displacement in quasi-static modelling for CSP. 


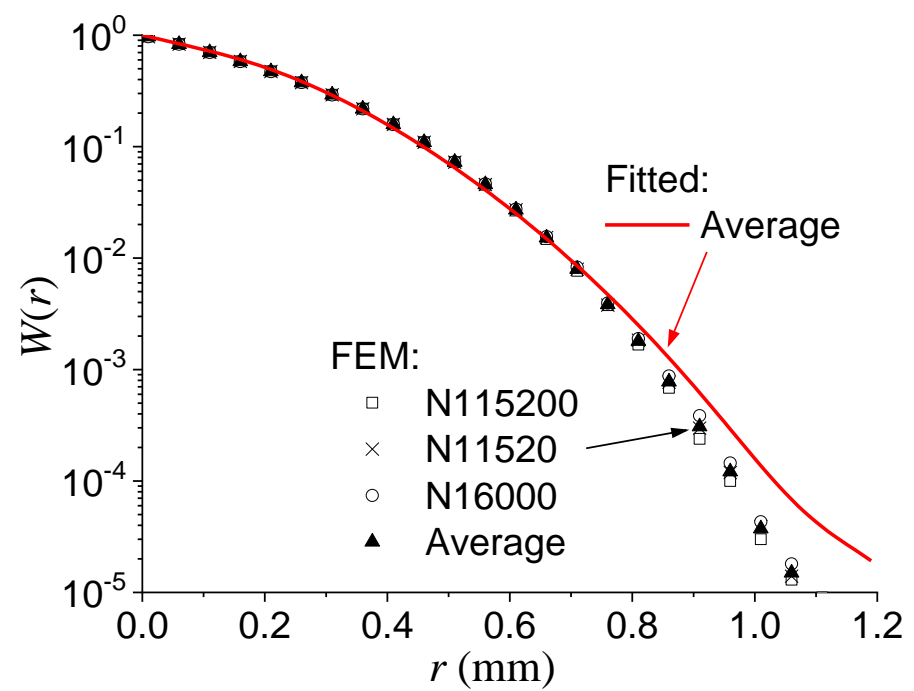

Figure 4. (Color online) Measured TPC data (points) from three 3D FEM material models (N115200, N11520, and N16000); the averages are shown by solid triangles. These averaged points are fitted by generalized TPC series (solid line), whose coefficients are given in Table IV. For better visualization, only every fifth measured data point of the measured data points are shown in the figure. This TPC has been obtained by 1 million random spatial samples of each of the three material domains. The samples were taken from within the body of the material, avoiding grains adjacent to the domain boundaries. 


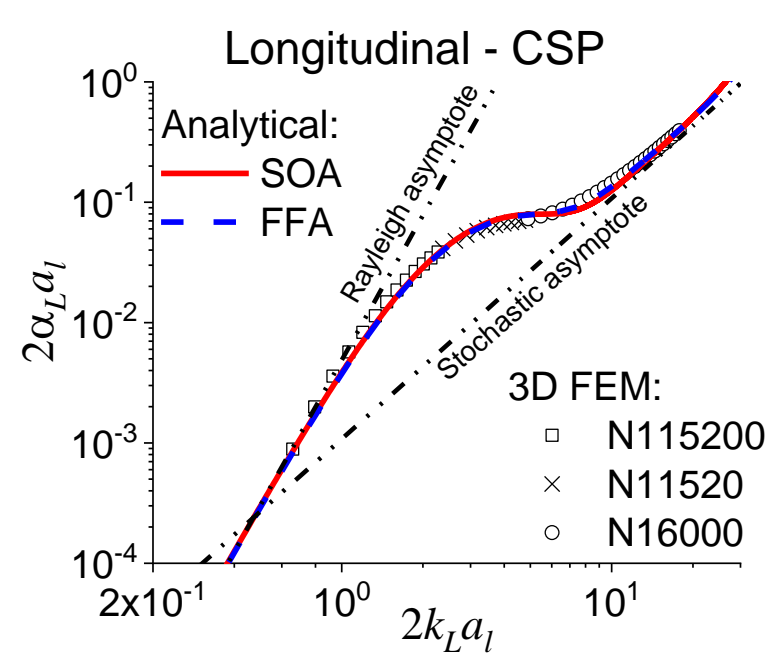

(a)

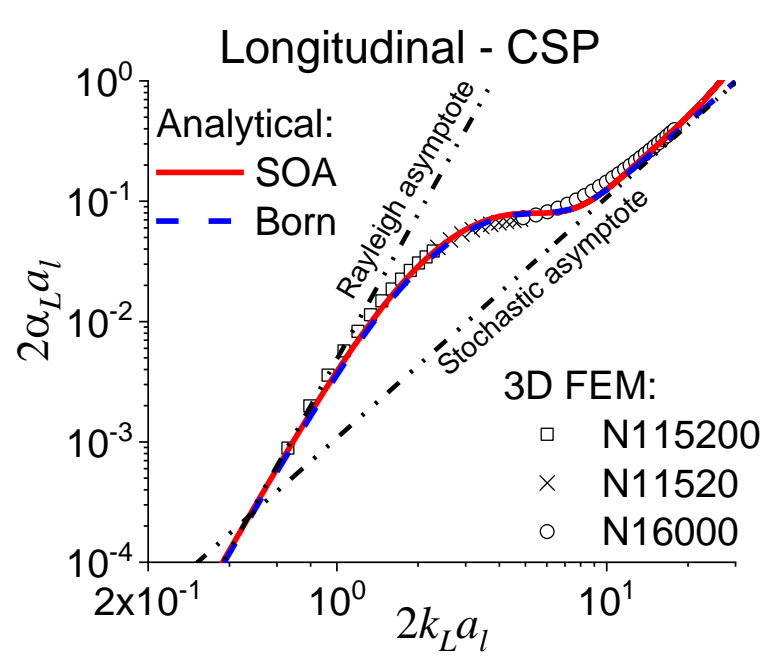

(b)

Figure 5. (Color online) Normalized longitudinal attenuation coefficient in polycrystalline

CSP versus nondimensional frequency $2 k_{L} a_{l}$ ( $a_{l}$ is mean line intercept). The 3D FEM attenuation is shown by points for the three material models; for better visualization the FEM data points are shown every ten points. The attenuation is computed by analytical models with the generalized TPC: the solid line is for the SOA and the dashed line is for the FFA in Figure (a), and the Born approximation in Figure (b). The Rayleigh and stochastic asymptotes are also shown. 


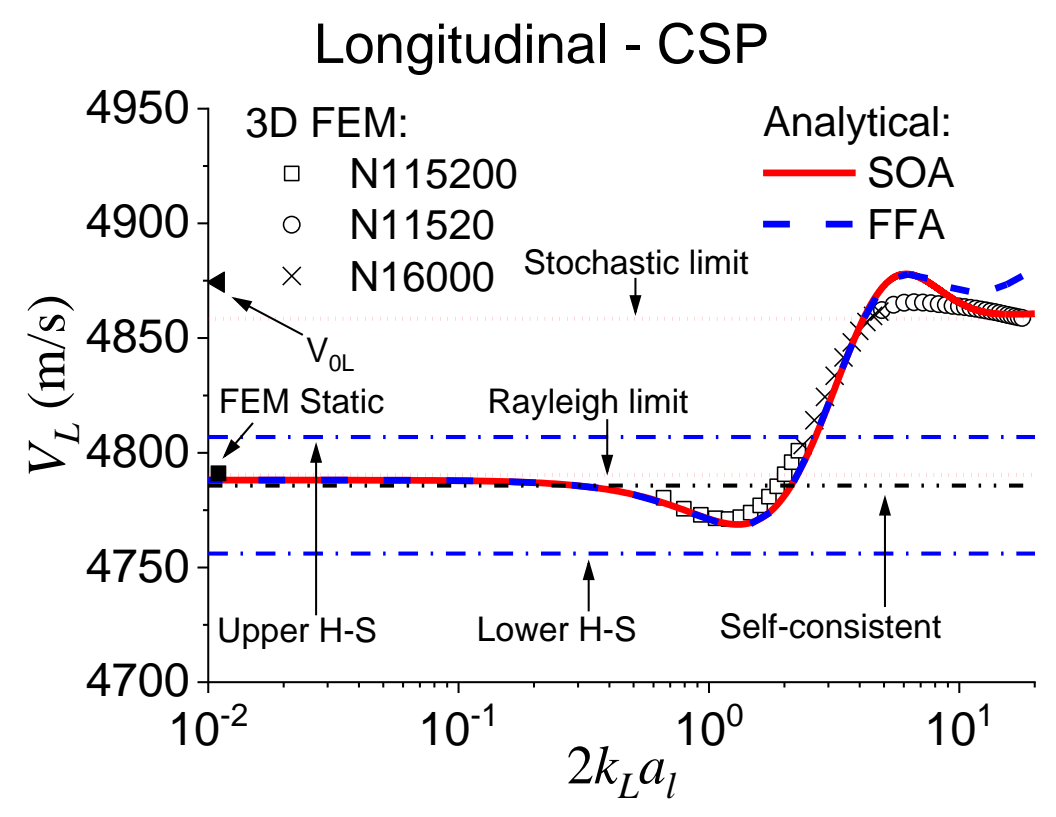

Figure 6. (Color online) Longitudinal phase velocity in polycrystalline CSP versus dimensionless frequency $2 k_{L} a_{l}$. 3D FEM velocity is shown by points; they are displayed every ten points for better visualization. The phase velocity from the analytical models with the generalized TPC, are shown by the solid line for the SOA model and the dashed line for the FFA model; the latter is used with the correction, Eq. (15). The FEM quasi-static velocity, the solid square dot, is shown to fit the scale at $2 k_{L} a_{l}=0.01$, instead of zero. Dotted horizontal lines show Rayleigh (Eq. (15)) and stochastic (Eq. (21)) phase velocity asymptotes. The Hashin-Shtrikman bounds (H-S Upper and H-S Lower) and the static velocity from the self-consistent static estimate (SC Static) are determined and shown. The Voigt velocity $V_{0 L}$ (the solid half triangle dot) is significantly above the upper H-S bound and even the stochastic asymptote. 


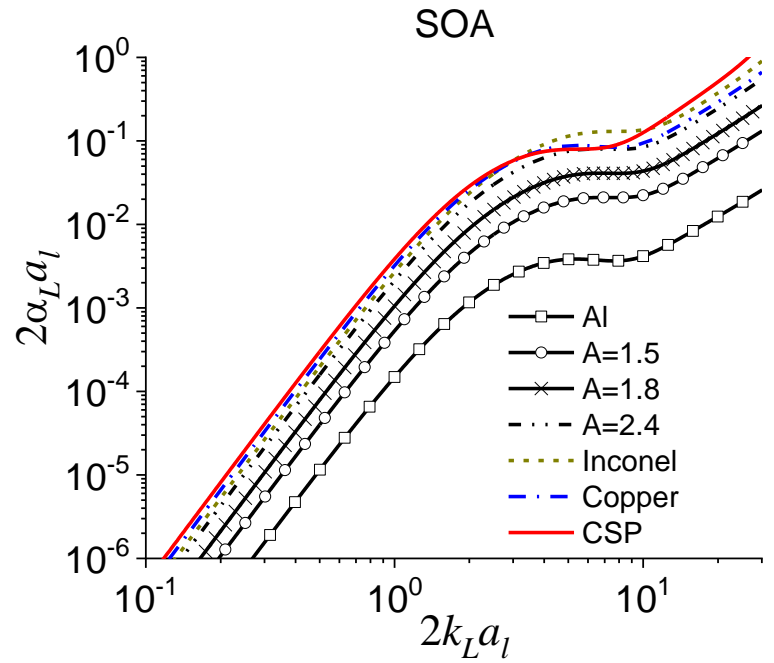

(a)

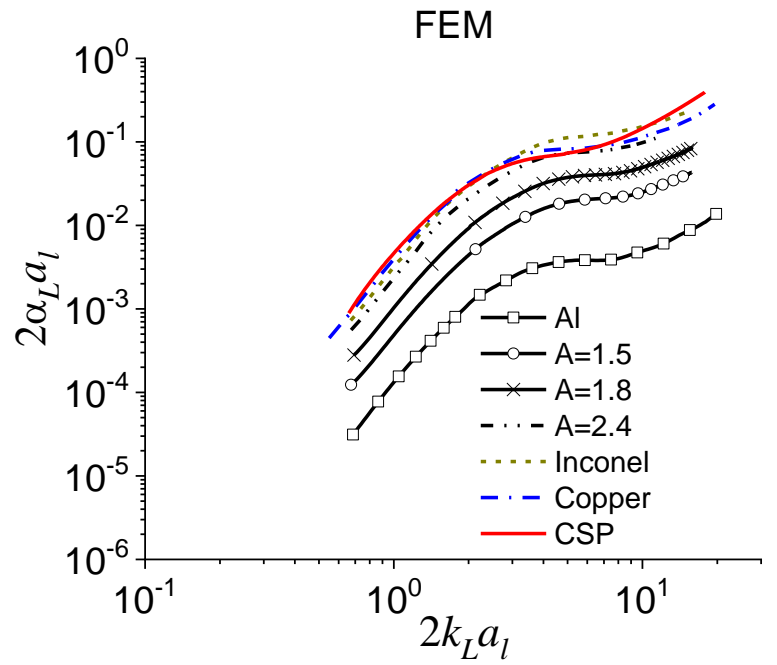

(b)

Figure 7. (Color online) Comparison of attenuation coefficients for all material systems that have been studied in our previous work [21,23] and this work: (a) attenuation curves from SOA and (b) FEM results. Each material system is normalized by its corresponding mean line intercept $a_{l}$. 


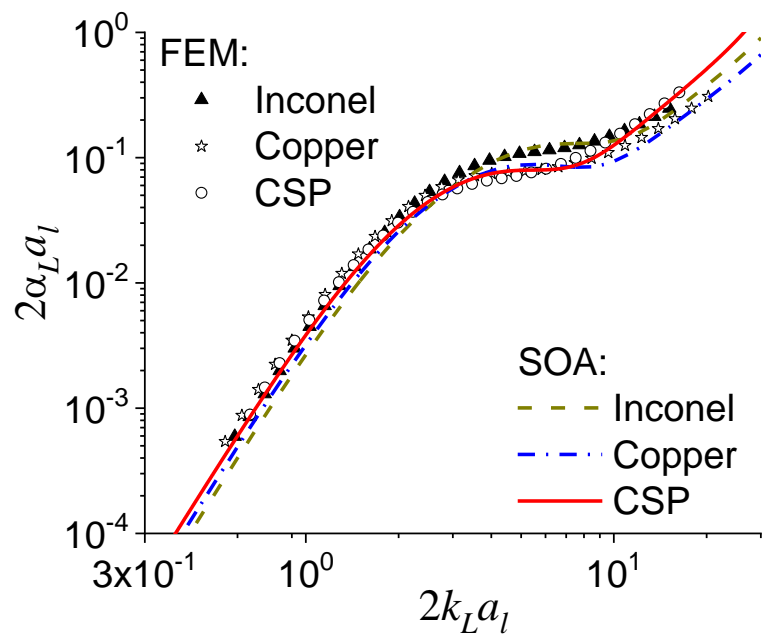

(a)

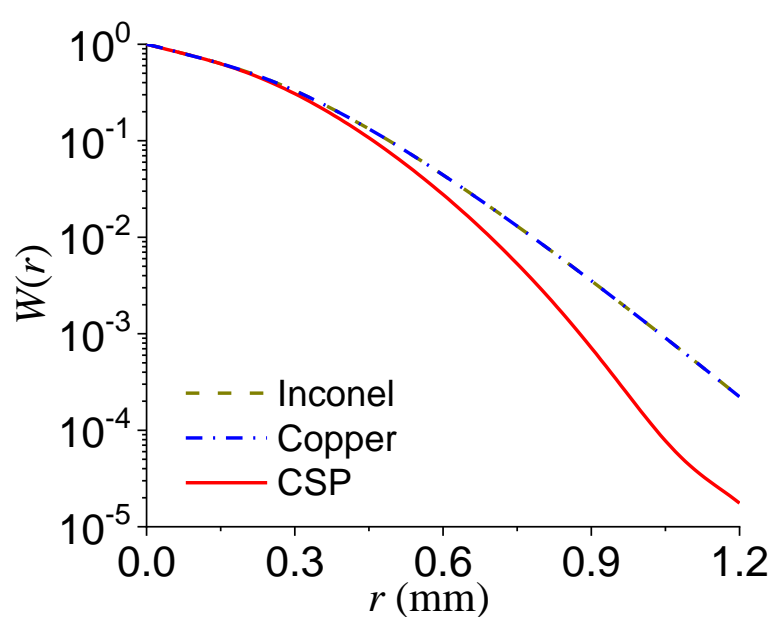

(b)

Figure 8. (Color online) (a) Comparison of longitudinal attenuation coefficients for Inconel, copper and CSP (SOA model- lines and the FEM results- points). Only part of the FEM data points is shown for better visualization. (b) Fitted TPC functions for Inconel, copper and CSP (all TPC functions are determined from the FEM material systems). 


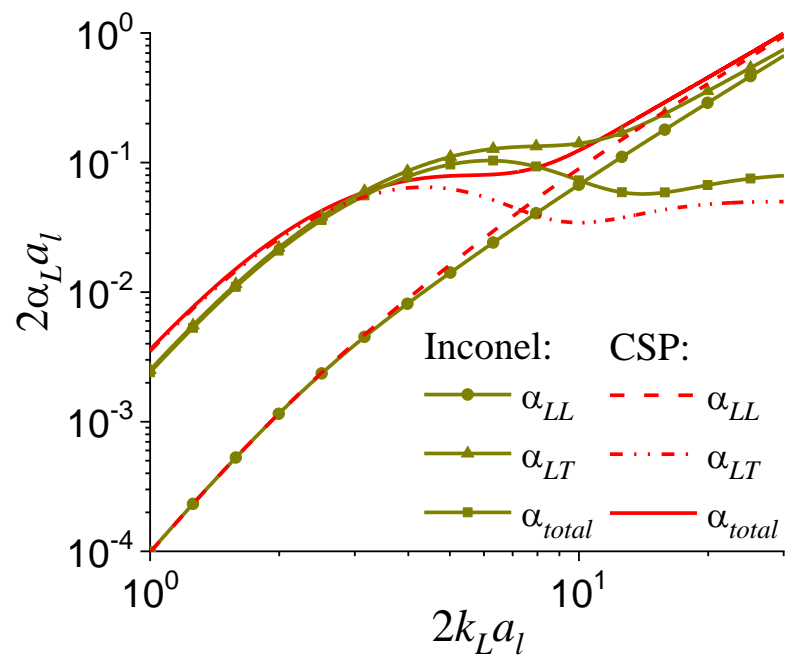

Figure 9 (Color online) Total attenuation $\alpha_{\text {total }}$ and its components $\alpha_{L T}, \alpha_{L L}$ calculated by Born approximation for the CSP and Inconel (obtained with the generalized TPC functions in Figure 8(b). 